\title{
La prise en charge des donneurs d'organes au Canada : recommandations du forum Optimiser le maintien du donneur afin d'accroître le nombre d'organes admissibles pour la transplantation
}

\author{
Sam D. Shemie, Heather Ross, Joe Pagliarello, Andrew J. Baker, Paul D. Greig, Tracy Brand, Sandra \\ Cockfield, Shaf Keshavjee, Peter Nickerson, Vivek Rao, Cameron Guest, Kimberly Young, \\ Christopher Doig, au nom du Groupe de recommandations en pédiatrie
}

$\mathrm{E}$ n collaboration avec la Société canadienne de soins intensifs, l'Association canadienne de transplantation et la Société canadienne de transplantation, le Conseil canadien pour le don et la transplantation (CCDT) a parrainé un forum intitulé "Optimiser le maintien du donneur afin d'accroître le nombre d'organes admissibles pour la transplantation", qui s'est tenu du 23 au 25 février 2004, afin d'élaborer des lignes directrices et des recommandations sur la prise en charge des donneurs d'organes au Canada. Les discussions ont été limitées à la période de soin qui commence par le diagnostic du décès neurologique (DDN), communément appelé "mort cérébrale», et le consentement à un don d'organes et se termine par le prélèvement chirurgical des organes. Cette période offre une importante possibilité d'optimiser la fonction de multiples organes et d'en améliorer l'utilisation.

Le forum était la première activité structurée coopérative à réunir des professionnels de la santé œuvrant dans les domaines des soins intensifs et de la transplantation et peut être considéré comme un point tournant au Canada. Les soins en fin de vie dispensés à l'unité des soins intensifs comprennent toutes les mesures visant à concrétiser la volonté et la possibilité de faire un don d'organes. Il faut conjuguer à une telle concertation dynamique qui vise à établir les meilleures pratiques de prise en charge des donneurs aux soins intensifs et à la salle d'opération des stratégies d'utilisation optimale des organes qu'il faut à leur tour relier à l'amélioration des transplantations et aux résultats chez les patients.

\section{Aperçu du Forum}

Les traitements de protection des organes reposent sur une prise en charge clinique experte visant à augmenter le nombre des donneurs admissibles et à optimiser la fonction des organes destinés à des transplantations. Les experts qui ont participé au forum ont concentré leur attention sur deux aspects clés :

comment optimiser la physiologie des organes de donneurs;

- comment augmenter le nombre de donneurs possibles dont les organes conviennent pour un don, augmenter le nombre d'organes transplantés par donneur, améliorer le fonctionnement et la survie du greffon ainsi que la survie du patient, et déterminer et explorer les défis logistiques qui se posent entre le diagnostic de décès neurologique (DDN) et le prélèvement des organes.

Cette initiative ne portait pas sur les défis connexes, comme le processus d'obtention du consentement, la perfusion des organes après le prélèvement, ainsi que la logistique de l'équipe chargée du prélèvement chirurgical ou de la conservation.

Le forum visait à :

- examiner et comparer les pratiques, les lignes directrices et les politiques nationales et internationales en vigueur sur la prise en charge des organes de donneurs, y compris les traitements de protection (les sources comprenaient notamment des articles et des rapports publiés dans des revues de sciences fondamentales et dans des publications cliniques, des lignes directrices régionales, nationales et internationales sur la prise en charge des donneurs, ainsi que des documents de conférences et d'ateliers sur le sujet);

- formuler des recommandations issues d'un consensus d'experts sur les traitements nécessaires pour protéger les organes aux soins intensifs et prendre en charge le donneur à la salle d'opération;

- formuler des recommandations issues d'un consensus d'experts à l'intention du CCDT, de la Société canadienne de transplantation, de la Société canadienne de soins intensifs, de l'Association canadienne de transplantation et d'autres organismes et groupes concernés;

- élaborer un mécanisme de liaison entre le milieu des soins intensifs et celui des transplantations afin de réviser et de mettre à jour les recommandations issues d'un consensus d'experts à mesure que les traitements évoluent;

- diffuser les résultats du Forum à la lumière de la compréhension actuelle de l'application des connaissances au Canada;

- élaborer des recommandations pour de futures recherches dans ce domaine en évolution constante.

Les participants étaient des professionnels de la santé provenant de 27 organismes, y compris des spécialistes des soins intensifs aux adultes et aux enfants, des médecins et chirurgiens spécialisés en transplantation d'organes chez les adultes et les enfants, des neurologues, des neurochirurgiens, des anesthésistes, des urgentologues, des infirmières et des 
infirmières praticiennes. Un groupe de travail composé d'administrateurs de services de santé, de responsables de politiques et de représentants d'organismes de coordination des dons et transplantations a aussi apporté sa contribution au sujet des obstacles et appuis logistiques et sur l'application des connaissances pour favoriser une prise en charge médicale efficace des donneurs. Les discussions ont visé avant tout un processus décisionnel basé sur la concertation et la collaboration, à un échelon stratégique national.

Ces lignes directrices s'adressent principalement aux médecins (ou à leurs délégués) qui interviennent dans la prise en charge de donneurs d'organes aux soins intensifs et à la salle d'opération. On y aborde les cinq domaines suivants :

- prise en charge multisystémique de donneurs d'organes multiples;

- considérations spécifiques aux organes dans le cas du cœur, des poumons et des organes abdominaux;

- autres défis systémiques;

- questions que l'on pourrait poser pour un programme national de recherche visant à optimiser la prise en charge des organes de donneurs;

- considérations relatives à la logistique et à l'application des connaissances ayant trait à la diffusion et à la mise en œuvre des recommandations du forum.

\section{Processus}

Les nombreux documents de fond fournis par le comité directeur avant le forum comportaient des recensions détaillées d'écrits et des enquêtes sur la pratique connexe. Au cours du forum, les participants ont abordé chacun des cinq domaines en procédant de la façon décrite ci-après. Des plénières ont suivi les exposés d'experts. Les participants ont ensuite travaillé en atelier guidés par les feuilles de travail contenant une description des soins courants et bien acceptés dans le contexte canadien (fondée sur une analyse des lignes directrices relatives à la prise en charge de donneurs en vigueur dans les établissements de santé du Canada ${ }^{1}$ ), un résumé des données scientifiques existantes, des considérations clés, un résumé des lignes directrices nationales et internationales sur la prise en charge de donneurs et une liste de références.

Les ateliers ont porté avant tout sur les détails des processus de soins. Le Groupe des recommandations issues du Forum (GRF) et le Groupe des recommandations applicables aux enfants (GRE) se sont réunis pour analyser les résultats des ateliers et des plénières et pour élaborer des recommandations pour les adultes et les enfants. Ces recommandations ont fait l'objet de nouvelles discussions au cours d'une plénière. On a recueilli et résumé les questions de recherche proposées par les participants (Annexe I). Le Groupe de la logistique et de l'application des connaissances (LAC) a étudié les questions relatives à la logistique et au partage des connaissances qui ont été soulevées durant le Forum (Annexe 2).

\section{Thèmes dominants}

Plusieurs thèmes dominants sont ressortis des discussions :

- Des recherches prospectives s'imposent pour améliorer la base des niveaux de preuve que les experts utilisent actuellement pour dégager des consensus sur les normes de soins. La plupart des données existantes sur la prise en charge des donneurs reposent sur des études prospectives, non contrôlées et d'observation.

- En raison de l'hypertension intracrânienne non contrôlée et de l'herniation cérébrale, le donneur d'organes présente une pathophysiologie distincte.

- En raison des variations temporelles du fonctionnement de multiples organes après le DDN, la détermination du moment optimal de prélèvement exige de la souplesse. Les participants ont établi que :

- la réanimation cardiorespiratoire est bénéfique pour la fonction de tous les organes;

- il importe de prendre le temps, aux soins intensifs, d'optimiser la fonction de multiples organes afin d'améliorer les résultats des transplantations;

- il est possible de réduire la dysfonction réversible des organes par une réanimation énergique et des réévaluations fréquentes;

- lorsque la fonction des organes est optimisée, il faut les prélever d'urgence.

- Une étude de l'utilisation ${ }^{2}$ des cœurs et des poumons dans quatre centres canadiens a permis de relever des lacunes possibles dans le processus visant à obtenir le consentement pour le don d'un seul organe, l'offre d'organes et l'utilisation des organes offerts. Le Forum recommande qu'il n'y ait aucun facteur démographique ni seuil de dysfonction prédéterminé qui empêche de consentir au don d'un seul organe ou d'offrir des organes pour la transplantation.

- Les décisions finales quant à la transplantabilité incombent encore à chaque programme de transplantation, représenté par les médecins chargés de la transplantation d'organes particuliers.

- Il faut évaluer les initiatives visant à accroître le nombre éventuel d'organes de donneurs en fonction non seulement d'une augmentation de l'utilisation des organes, mais aussi des résultats des transplantations correspondantes.

- La collaboration entre les soins intensifs et l'équipe de transplantation à cet égard vise à assurer une responsabilisation réciproque des services de prélèvement et de transplantation si l'on n'utilise pas les organes disponibles.

\section{Prise en charge multisystémique du donneur d'organes multiples}

L'Annexe 3 résume les règlements sur la prise en charge des donneurs.

\section{Hypertension artérielle générale liée à la pression intracrânienne}

\section{Recommandation 1.1 : Seuils et thérapie privilégiée}

Nous recommandons que l'hypertension artérielle qui se déclare après le diagnostic de décès neurologique (DDN) soit traitée ainsi : 
Seuils :

- tension artérielle systolique > $\mathrm{r} 60 \mathrm{~mm} \mathrm{Hg}$ et (ou)

- tension artérielle moyenne $>90 \mathrm{~mm} \mathrm{Hg}$

Traitement privilégié :

- nitroprussiate, à une dose de o,5 à 5,0 $\mu \mathrm{g} / \mathrm{kg} / \mathrm{min}$, et (ou)

- esmolol, à une dose de Ioo à $500 \mu \mathrm{g} / \mathrm{kg}$ en bol, suivie d'une dose de roo à $300 \mu \mathrm{g} / \mathrm{kg} / \mathrm{min}$

Il faut titrer les doses perfusées jusqu'à ce que l'on obtienne l'effet clinique désiré.

\section{Pratique actuelle au Canada}

Il existe des variations importantes dans la pratique.

\section{Considérations clés}

- Il faut établir une distinction entre la tempête catécholaminergique liée à la pression incrânienne aiguë et l'hypertension artérielle qui peut se manifester durant l'herniation cérébrale, mais avant le DDN. Le Forum n'a pas abordé cette période de soins.

- Compte tenu des changements constants et du risque de détérioration de la fonction cardiovasculaire après le DDN, il est préférable d'employer des agents à action brève.

- On peut administrer d'autres médicaments comme :

- la nitroglycérine pour réduire (par ex.: le risque de vol coronarien par rapport au nitroprussiate);

- le labétolol, plus couramment disponible et utilisé que l'esmolol dans la pratique clinique; sa demi-vie biologique prolongée (de 4 à 6 heures) préoccupe toutefois.

- L'hypertension observée pendant l'établissement de l'utilisation d'agents vasopresseurs ou d'un soutien inotrope indique qu'il faut réduire ce soutien au lieu de commencer à administrer des antihypertenseurs.

\section{Performance cardiovasculaire, surveillance et soutien hémodynamique}

\section{Considérations globales}

La détérioration de la fonction cardiovasculaire associée à l'hypertension intracrânienne, caractérisée par la tempête sympathique qui entraîne une dysfonction neurogénique du myocarde ${ }^{3}$ et une vasoconstriction intense suivie d'un épuisement sympathique, et une vasodilatation ${ }^{4}$ variera en fonction des facteurs suivants :

- Rapidité de l'élévation de la pression intracrânienne $e^{5}$

- Temps écoulé après l'herniation cérébrale

- Étiologie de l'atteinte cérébrale (par ex.: contusion myocardique traumatique, ischémie consécutive à un arrêt cardiaque ou à un état de choc, hypoxémie).

Il est reconnu que les intensivistes titrent le traitement cardiovasculaire en fonction de cibles cliniques, biochimiques et hémodynamiques permettant de rétablir le volume intravasculaire sans hypervolémie et d'assurer un soutien approprié du myocarde et du système vasculaire pour obtenir un débit cardiaque optimal pour la perfusion des organes.

La mise en œuvre de mesures de soutien cardiovasculaire présuppose qu'on a rétabli la normovolémie chez les patients.

L'évaluation de l'état cardiocirculatoire oblige à évaluer de multiples variables. Un traitement ne doit pas dépendre d'une seule mesure ni valeur prise isolément.

Il faut conjuguer l'intensification des mesures de soutien à un resserrement de la surveillance hémodynamique.

Même si ces valeurs cibles servent à guider le traitement, il faut établir un équilibre entre leur application rigoureuse et l'évaluation globale de l'état cardiovasculaire effectuée par des cliniciens expérimentés.

Les mesures de soutien cardiovasculaire doivent reposer sur une physiologie rationnelle. Il faut distinguer les vasopresseurs purs (vasopressine, phényléphrine) des vasopresseurs qui ont des effets inotropes sur les bêta-agonistes (norépinéphrine, épinéphrine). La prudence s'impose si l'on administre un traitement aux bêta-agonistes à des donneurs éventuels de cœur en raison des risques d'épuisement des réserves d'adénosine triphosphate (ATP) dans le myocarde et de la régulation négative des récepteurs bêta ${ }^{6}$. Si l'on envisage le don du cœur, la dose de dopamine ou de son équivalent ne doit pas dépasser Io $\mu \mathrm{g} / \mathrm{kg} / \mathrm{min}$.

\section{Pratique actuelle au Canada}

Les mesures suivantes sont couramment acceptées dans la pratique et sont approuvées a priori :

- surveillance standard : ligne artérielle, ligne veineuse centrale, électrocardiogramme à 12 dérivations;

- cibles hémodynamiques recommandées comprenant :

- tension artérielle moyenne de $\geq 70 \mathrm{~mm} \mathrm{Hg}$

- tension artérielle systolique de $\geq 100 \mathrm{~mm} \mathrm{Hg}$, fréquence cardiaque de 60 à 120 battements/minute

- pression veineuse centrale de 6 à Io mm Hg (normovolémie);

- les mesures standard de soutien inotrope comprennent notamment la dopamine à une dose $\mathrm{de} \leq \mathrm{Io} \mu \mathrm{g} / \mathrm{kg} / \mathrm{min}$ (ou l'équivalent).

\section{Recommandation 2.1 : Surveillance de la saturation en oxygène centrale ou du sang veineux mêlé}

Une surveillance de la saturation en oxygène du sang veineux mêlé est indiquée chez les patients qui présentent une instabilité hémodynamique continue. Le traitement hémodynamique doit viser une cible de $\geq 60 \%$.

\section{Pratique actuelle au Canada}

Il n'existe pas de données à ce sujet.

\section{Considérations clés}

- Les tendances sériées sont plus utiles que des mesures uniques.

- L'oxymétrie du sang veineux mêlé peut être déterminée au moyen d'échantillons prélevés de façon intermittente dans l'artère pulmonaire ou de façon continue par des cathéters oxymétriques.

- L'extraction d'oxygène tissulaire n'a pas été bien étudiée chez des patients en état de décès neurologique. Des valeurs faibles peuvent indiquer un apport réduit d'oxygène. L'interprétation de valeurs élevées n'est toutefois pas fiable en présence d'un arrêt de la fonction neurologique ou de la circulation cérébrale. 
- L'oxymétrie du sang veineux central n'a pas été bien étudiée chez des patients en état de décès neurologique.

\section{Recommandation 2.2 : Surveillance du lactate}

Nous recommandons que le taux de lactate soit déterminé par des mesures sériées chez tous les patients. Si les taux de lactate sont élevés ou à la hausse, nous recommandons une investigation visant à déterminer l'étiologie.

Pratique actuelle au Canada

Il n'existe pas de données à ce sujet.

\section{Considérations clés}

Des taux de lactate à la baisse sont un signe d'amélioration de la distribution d'oxygène.

\section{Recommandation 2.3 : Indications pour l'emploi d'un cathéter de l'artère pulmonaire}

Nous recommandons l'emploi d'un cathéter de l'artère pulmonaire (CAP) dans les cas suivants :

- La fraction d'éjection évaluée par échocardiographie bidimensionnelle est de $\leq 40 \%$, ou

- les patients ont besoin

- de dopamine (> Io $\mu \mathrm{g} / \mathrm{kg} / \mathrm{min}$ ) ou l'équivalent,

- d'un traitement de soutien par vasopresseur (exclut la vasopressine comme élément d'une hormonothérapie) et (ou)

- d'une intensification des mesures de soutien.

Les valeurs hémodynamiques visées lorsqu'on utilise un CAP sont les suivantes : pression capillaire pulmonaire (PCP) de 6 à $\mathrm{I} 0 \mathrm{~mm} \mathrm{Hg}$; index cardiaque de $>2,4$ litre $/ \mathrm{min} / \mathrm{m}^{2}$; résistance vasculaire systémique (RVS) de 800 à I200 dynes $/ \mathrm{sec} / \mathrm{cm}^{5}$; index de travail systolique du ventricule gauche : > $15 \mathrm{~g} / \mathrm{kg}$-min.

\section{Pratique actuelle au Canada}

Il existe des variations importantes dans la pratique.

\section{Considérations clés}

- Le terme «vasopresseur» désigne un agent vasoconstricteur.

- Même si l'utilisation d'un CAP dans la pratique des soins intensifs chez les adultes est à la baisse, le donneur d'organe présente une pathophysiologie distincte.

- L'emploi d'un CAP est justifié non seulement pour préciser le titrage du soutien hémodynamique, mais aussi pour évaluer la transplantabilité du cœur et des poumons ${ }^{7}$.

- L'échocardiographie bidimensionnelle est indiquée principalement pour évaluer la fonction cardiaque et savoir si le cœur peut être transplanté. Le rôle d'échocardiographies uniques ou en série dans l'évaluation de la fonction cardiaque qui vise à orienter le traitement hémodynamique chez un donneur d'organes instable n'est pas bien établi.

Recommandation 2.4 : Agents de première intention pour le soutien hémodynamique - vasopressine

Nous recommandons d'utiliser la vasopressine comme traite- ment de soutien hémodynamique lorsque des agents vasopresseurs sont indiqués. La dose maximale doit être de 2,4 U/h (o,04 U/min).

Pratique actuelle au Canada

Il existe des variations importantes dans la pratique.

\section{Considérations clés}

- La vasopressine est un agent particulier, parce qu'elle peut servir à diverses applications, comme le soutien vasopresseur hémodynamique, le traitement du diabète insipide et l'hormonothérapie.

- Il faut normaliser les unités d'administration de doses.

- Le sevrage des catécholamines de soutien est la première stratégie à suivre pour traiter l'hypertension artérielle chez les patients traités à la vasopressine.

\section{Recommandation 2.5 : Agents de deuxième intention} pour le soutien hémodynamique - norépinéphrine, épinéphrine et phényléphrine

Nous recommandons d'utiliser la norépinéphrine, l'épinéphrine et la phényléphrine comme mesures de soutien hémodynamique. Il faut ajuster le traitement de façon à produire l'effet clinique désiré, sans dose maximale préétablie.

Pratique actuelle au Canada

Il existe des variations importantes dans la pratique.

\section{Considération clé}

Il faut augmenter les doses de catécholamines en fonction des valeurs obtenues par CAP. La prudence est de rigueur lorsque l'on administre un de ces agents à des doses de plus de $0,2 \mu \mathrm{g} / \mathrm{kg} / \mathrm{min}$.

\section{Glycémie et nutrition}

\section{Recommandation 3.1 : Maîtrise de la glycémie}

Nous recommandons de contrôler la glycémie par des perfusions d'insuline titrées de façon à maintenir une glycémie de 4 à $8 \mathrm{mmol} / \mathrm{L}$.

\section{Pratique actuelle au Canada}

Il existe des variations importantes dans la pratique.

\section{Considération clé}

Il ne faut pas interpréter à tort le recours à l'insuline comme une insulinodépendance qui pourrait empêcher la transplantation de cellules des îlots de Langerhans. Si une précision s'impose, il faut mesurer les taux d'hémoglobine (HgB) $A_{1 C}$ dans ces circonstances.

\section{Recommandation 3.2 : Nutrition}

- Il faut administrer de routine des perfusions intraveineuses de dextrose.

- Il faut entreprendre ou continuer une alimentation entérale de routine, selon la tolérance, et y mettre fin 
lorsqu'on appelle le donneur à la salle d'opération.

- Il ne faut pas entreprendre d'alimentation parentérale, mais il faut toutefois la maintenir lorsqu'elle est déjà en place.

Pratique actuelle au Canada

Il n'existe pas de données à ce sujet.

\section{Considérations clés}

Aucune.

\section{Diabète insipide et hypernatrémie}

\section{Pratique actuelle au Canada}

Les mesures suivantes sont des aspects couramment acceptés dans la pratique et sont approuvées a priori :

- Sodium sérique : valeurs visées entre 130 et $150 \mathrm{mmol} / \mathrm{L}$.

- Débit urinaire : plage visée de 0,5 à $3 \mathrm{~mL} / \mathrm{kg} / \mathrm{h}$ (chez les adultes et les enfants).

- On peut définir le diabète insipide comme un débit urinaire de $>4 \mathrm{~mL} / \mathrm{kg} / \mathrm{h}$ chez les adultes et les enfants.

- Associé à une montée du sodium sérique $(\geq \mathrm{I} 45 \mathrm{mmol} / \mathrm{L}$ ).

- Associé à une montée de l'osmolarité sérique $(\geq 300 \mathrm{mOsm})$ et à une baisse de l'osmolarité urinaire $(\leq 200 \mathrm{mOsm})$.

- Posologie de la I-désamino-D-arginine vasopressine (DDAVP) contre le diabète insipide :

- Adultes : I-4 $\mu \mathrm{g}$ par voie intraveineuse (IV) et ensuite I-2 $\mu \mathrm{g}$ IV aux six heures pour produire un débit urinaire de $<4 \mathrm{~mL} / \mathrm{kg} / \mathrm{h}$

- Enfants : 0,25-I $\mu$ g par voie IV aux six heures pour produire un débit urinaire de $<4 \mathrm{~mL} / \mathrm{kg} / \mathrm{h}$.

\section{Recommandation 4.1 : Diabète insipide}

- Il est possible de traiter le diabète insipide seul par une perfusion IV continue de vasopressine $(\leq 2,4 \mathrm{U} / \mathrm{h})$ ou une perfusion IV intermittente de DDAVP.

- Dans les circonstances suivantes, la perfusion de vasopressine doit être le traitement de première intention :

- soutien hémodynamique avec vasopressine nécessaire,

- hormonothérapie combinée commencée.

- Il faut utiliser au besoin la DDAVP comme supplément à la vasopressine.

- Il n'est pas nécessaire d'interrompre l'administration de DDAVP avant l'arrivée du donneur à la salle d'opération.

\section{Considération clé}

La DDAVP est un analogue de l'AVP qui a un effet antidiurétique relativement pur et une activité vasopressive négligeable ${ }^{8}$. Les limites supérieures de la posologie de DDAVP sont empiriques. Il n'y a pas de limite supérieure claire à la dose de DDAVP, car il faut la titrer de façon à obtenir l'effet visé, qui est de réduire le débit urinaire.

\section{Recommandation 4.2 : Hypernatrémie}

Nous recommandons de traiter l'hypernatrémie chez tous les donneurs qui présentent des taux de sodium sérique de plus de $150 \mathrm{mmol} / \mathrm{L}$.

\section{Considération clé}

Il y a un lien indépendant entre l'hypernatrémie (sodium sérique > $155 \mathrm{mmol} / \mathrm{L}$ ) et la dysfonction hépatique et la perte du greffon'. Outre le contrôle du sodium, il faut normaliser empiriquement la concentration de calcium, de phosphate, de potassium et de magnésium.

\section{Hormonothérapie combinée}

\section{Recommandation 5.1 : Hormone thyroïdienne, vasopressine et méthylprednisolone}

L'hormonothérapie combinée consiste à administrer :

- de l'hormone thyroïdienne (tétra-iodothyronine ou $\mathrm{T}_{4}$ ), bol de $20 \mu \mathrm{g}$ par voie IV suivi d'une perfusion IV de Io $\mu \mathrm{g} / \mathrm{h}$,

- de la vasopressine, bol IV de I U suivi d'une perfusion IV de $2,4 \mathrm{U} / \mathrm{h}$,

- de la méthylprednisolone, $15 \mathrm{mg} / \mathrm{kg}$ IV aux 24 heures.

Nous recommandons une hormonothérapie combinée chez les donneurs qui présentent une fraction d'éjection de $\leq 40 \%$, évaluée par électrocardiographie bidimensionnelle, ou une instabilité hémodynamique. Il faut envisager d'y recourir chez tous les donneurs.

\section{Pratique actuelle au Canada}

Il existe des variations importantes dans la pratique.

\section{Considérations clés}

- L'instabilité hémodynamique inclut un état de choc qui ne répond pas aux tentatives de rétablissement d'une normovolémie et nécessite un soutien vasoactif (dopamine à une dose de > Io $\mu \mathrm{g} / \mathrm{min}$ ) ou l'administration d'un vasopresseur.

- Tirées d'une étude de cohortes rétrospective d'envergure réalisée aux États-Unis par le United Network for Organ Sharing (UNOS $)^{10}$, les données existantes semblent indiquer qu'une hormonothérapie triple offre des avantages importants et présente des risques minimes. Une analyse de régression logistique à variables multiples des données recueillies chez I8 726 donneurs en état de mort cérébrale a révélé une hausse importante des taux d'utilisation des reins, des foies et des cœurs prélevés chez des donneurs qui ont reçu une triple hormonothérapie. Elle révèle aussi des améliorations importantes de la survie d'une greffe de rein à un an et de la survie des patients qui ont reçu un cœur. On n'a pas effectué d'essai randomisé prospectif.

- Puisque la conversion de la $\mathrm{T}_{4}$ en tri-iodothyronine $\left(\mathrm{T}_{3}\right)$ dans les tissus périphériques peut être déficiente chez les donneurs d'organes et chez les patients qui suivent une corticothérapie, on peut préférer les solutions de $\mathrm{T}_{3}$ intraveineuse qui n'est toutefois pas encore commercialisée au Canada. Des solutions intraveineuses de $\mathrm{T}_{4}$ et des préparations entérales de $\mathrm{T}_{3}$ sont actuellement disponibles. 
- Chez les patients de l'étude UNOS qui recevaient une hormonothérapie $^{10}$, on a utilisé des préparations de $\mathrm{T}_{4}$ dans $93 \%$ des cas et des solutions de $\mathrm{T}_{3}$ dans $6,9 \%$ des cas. Le nombre de patients n'était toutefois pas suffisant pour permettre de déceler un avantage de la T3 par rapport à la $\mathrm{T}_{4}$.

- Lorsque la $\mathrm{T}_{4}$ est administrée sous forme de perfusion intraveineuse continue, sa stabilité en solution et son adhérence possible à la tubulure de plastique en raison de sa nature hydrophobe peuvent en modifier la biodisponibilité. Les répercussions quantitatives de ce problème pharmacologique ne sont pas claires. Une autre possibilité consiste à administrer la $\mathrm{T}_{4}$ par voie intraveineuse, comme suit : bol de roo $\mu$ g par voie intraveineuse suivi d'une perfusion intraveineuse de $50 \mu \mathrm{g}$ aux $\mathrm{I}_{2}$ heures. Si des données futures montrent que la $\mathrm{T}_{3}$ intraveineuse offre des avantages par rapport à la $T_{4}$, il faudrait exercer des pressions auprès de la Direction générale de la protection de la santé de Santé Canada afin que la thérapie à la $T_{3}$ intraveineuse soit disponible au Canada pour cette indication.

- Il faut réunir des données sur l'absorption et la pharmacocinétique des préparations entérales de $\mathrm{T}_{3}$ chez les donneurs d'organes avant d'en recommander l'utilisation.

- Il faut commencer le traitement à la vasopressine à un débit fixe. Si le traitement provoque une hypertension artérielle, il faut procéder au sevrage des catécholamines avant de réduire le débit de la perfusion de vasopressine.

\section{Recommandation 5.2 : Corticostéroïdes et protection des poumons}

Nous recommandons d'administrer de la méthylprednisolone par voie intraveineuse à tous les donneurs, à une dose de $15 \mathrm{mg} / \mathrm{kg}$ aux 24 heures. Il faut amorcer le traitement après le DDN.

\section{Pratique actuelle au Canada}

On administre $15 \mathrm{mg} / \mathrm{kg}$ de méthylprednisolone par voie IV à des donneurs éventuels de poumon (dose maximale de I g).

\section{Considération clé}

Une corticothérapie est actuellement indiquée comme traitement immunomodulateur chez les donneurs possibles de poumons ${ }^{11}$, mais les protocoles d'administration de corticostéroïdes ne sont pas uniformes.

\section{Seuils transfusionnels}

\section{Recommandation 6.1 : Cibles acceptables pour les paramètres relatifs à l'hémoglobine, aux plaquettes et à la coagulation}

- Un taux d'hémoglobine cible de 90 à $100 \mathrm{~g} / \mathrm{L}$ est idéal pour optimiser la fonction cardiorespiratoire en présence d'une instabilité hémodynamique. Une concentration de $70 \mathrm{~g} / \mathrm{L}$ constitue la limite la plus basse acceptable pour la prise en charge de donneurs stables aux soins intensifs.

- Il n'y a pas d'objectifs précis quant à la concentration de plaquettes, au ratio international normalisé ou au temps de céphaline. Le remplacement des plaquettes ou du facteur plasmatique est indiqué en cas de saignement clinique seulement.

- Il faut prélever les échantillons de sang destinés aux analyses de sérologie et au typage tissulaire des donneurs avant les transfusions afin de minimiser le risque de faux résultats attribuables à une hémodilution.

- Les transfusions chez des donneurs d'organes ne nécessitent aucune précaution particulière.

\section{Pratique actuelle au Canada}

Il existe des variations importantes dans la pratique.

\section{Considérations clés}

- Il peut y avoir un lien entre les transfusions de globules rouges et une activation inflammatoire liée à l'âge du sang.

- Il faut tenir compte de l'effet d'épargne que les transfusions de globules rouges ont sur l'utilisation des cristalloïdes chez les donneurs éventuels de poumons présentant une infiltration alvéolocapillaire.

- Il peut être nécessaire de corriger la thrombocytopénie et les paramètres de la coagulation avant de pratiquer des interventions effractives qui posent un risque d'hémorragie.

- Il faut adapter à chaque donneur les transfusions peropératoires de globules rouges, de plaquettes et de facteurs plasmatiques administrées par des anesthésistes et par l'équipe chargée de la transplantation.

- Au Canada, le sang est systématiquement déleucocyté et le risque de transmission du cytomégalovirus (CMV) est négligeable. Il n'est pas nécessaire d'administrer du sang non infecté par le CMV à des donneurs non infectés par le CMV.

\section{Infections bactériennes invasives}

\section{Recommandation 7.1 : Hémocultures quotidiennes}

- Il faut procéder à une hémoculture initiale de base pour tous les donneurs et la répéter après 24 heures et au besoin.

- Les hémocultures positives ou les infections confirmées ne sont pas des contre-indications au don d'organes.

- Il faut entreprendre une antibiothérapie dans les cas d'infection confirmée ou présumée. La durée du traitement dépend de la virulence du micro-organisme en cause et est déterminée conjointement avec l'équipe de transplantation et les services des maladies infectieuses.

- On ne peut définir actuellement de durée minimale du traitement avant le prélèvement des organes.

Pratique actuelle au Canada

Le soin standard inclut des cultures d'urine et de spécimens aspirés par tube endotrachéal. 


\section{Considérations clés}

Aucune.

\section{Recommandation 7.2 : Antibiotiques à large spectre}

- Un traitement empirique aux antibiotiques à large spectre n'est pas indiqué pendant la période de prise en charge des donneurs d'organes aux soins intensifs.

- Il faut laisser à la discrétion de l'équipe chirurgicale les décisions sur l'usage périopératoire d'antibiotiques.

Pratique actuelle au Canada

Il n'existe pas de données à ce sujet.

\section{Considérations clés}

Aucune.

\section{Considérations relatives à certains organes : cœur, poumons et organes abdominaux}

\section{Cœur}

\section{Pratique actuelle au Canada}

Les mesures suivantes sont des aspects couramment acceptés dans la pratique et sont approuvées a priori :

- Il faut soumettre les donneurs dont le cœur pourrait éventuellement être transplanté à une évaluation de routine par électrocardiographie et échocardiographie bidimensionnelle.

\section{Recommandation 8.1 : Évaluation initiale de la fonction cardiaque}

Si l'évaluation initiale de la fonction cardiaque révèle une fraction d'éjection de $\leq 40 \%$ fondée sur une évaluation échocardiographique bidimensionnelle, le traitement optimal consiste à insérer un cathéter de l'artère pulmonaire et à établir une thérapie afin d'atteindre les objectifs hémodynamiques CAP énumérés dans la recommandation 2.3.

\section{Considérations clés}

- On a établi un lien entre l'atteinte des cibles CAP et les résultats favorables d'une transplantation ${ }^{7,12}$.

- Il faut procéder à l'échocardiographie initiale servant à évaluer le donneur du cœur seulement après la réanimation hémodynamique. Il faut envisager de répéter l'échocardiographie après 6 heures. Il faut procéder à une étude prospective de l'utilité d'échocardiographies en série.

- L'emploi d'un CAP est justifié non seulement pour préciser le soutien hémodynamique nécessaire, mais aussi pour évaluer la transplantabilité du cœur et des poumons. La décision de procéder à la transplantation doit tenir compte de ces recommandations. Une échocardiographie bidimensionnelle qui donne des résultats anormaux, suivie de données hémodynamiques favorables obtenues par CAP, constitue une évaluation acceptable et une échocardiographie de suivi n'est pas nécessaire.
- On recommande d'utiliser le CAP lorsque les services appropriés d'expertise technique et d'interprétation sont disponibles.

\section{Recommandation 8.2 : Concentrations de troponine}

Nous recommandons de mesurer les concentrations de troponine (I ou T) aux $\mathrm{I} 2 \mathrm{~h}$ comme méthode de surveillance standard des données tant cliniques que pronostiques.

\section{Considération clé}

Il ne faut pas se baser uniquement sur les concentrations de troponine pour rejeter l'usage possible de cœurs aux fins de transplantation.

\section{Recommandation 8.3 : Coronarographie}

- Une coronarographie est indiquée chez les donneurs qui présentent les caractéristiques suivantes :

- homme $>55$ ans ou femme $>60$ ans;

- homme $>40$ ans ou femme $>45$ ans présentant deux facteurs de risque (voir Considérations clés);

- présence de trois facteurs de risque ou plus à n'importe quel âge;

- antécédents de prise de cocaïne.

- Si le centre hospitalier a les installations nécessaires et que la coronarographie est indiquée, il faut toujours administrer ce test afin de documenter l'anatomie coronarienne pour appuyer la prise de décision.

- Il ne doit y avoir aucun seuil absolu d'obstruction de la lumière coronarienne; les décisions doivent être prises en fonction de l'état du receveur, de la fonction cardiaque et de la possibilité d'effectuer un pontage aortocoronarien ou une intervention coronarienne percutanée, comme la pose d'un stent, p. ex.

- L'impossibilité de procéder à une coronarographie ne doit pas empêcher la transplantation. En l'absence de coronarographie, on peut évaluer la possibilité de transplanter le cœur en fonction des critères suivants :

- fraction d'éjection de > 40 \% à l'évaluation échocardiographique bidimensionnelle;

- stabilité hémodynamique; ou

- vérification chirurgicale au moment du prélèvement.

- Le transfert du patient dans un centre hospitalier de prélèvement doté des installations nécessaires pour la coronarographie est une option qu'il faut envisager au cas par cas, avec le consentement de la famille du donneur.

\section{Considérations clés}

- Les facteurs de risque cardiovasculaire de coronaropathie qui ont un effet sur les résultats de la transplantation comprennent les suivants ${ }^{13,14}$ :

- tabagisme;

- hypertension;

- diabète;

- hyperlipidémie;

- indice de masse corporelle > 32;

- antécédents familiaux de la maladie; 
- antécédents de coronaropathie;

- ischémie révélée par l'électrocardiographie;

- réduction de la mobilité de la paroi antérolatérale révélée par l'échocardiographie;

- évaluation échocardiographique bidimensionnelle de la fraction d'éjection de $\leq 40 \%$.

- Pour minimiser le risque de néphropathie induite par un agent de contraste :

- il faut s'assurer que la volémie est normale;

- il faut prescrire de la N-acétylcystéine en prophylaxie à des doses de 600 à $1000 \mathrm{mg}$, par voie entérale, 2 fois par jour, la première dose étant administrée dès que l'angiographie est indiquée; on peut aussi administrer de la N-acétylcystéine par voie intraveineuse, à une dose de $150 \mathrm{mg} / \mathrm{kg}$ diluée dans $500 \mathrm{~mL}$ de soluté physiologique normal, pendant 30 minutes, immédiatement avant l'injection de l'agent de contraste, et ensuite à une dose de $50 \mathrm{mg} / \mathrm{kg}$ dans $500 \mathrm{~mL}$ de soluté physiologique normal pendant 4 heures;

- il faut procéder aux angiographies au moyen d'un agent de contraste radiologique à faible risque (non ionique, iso-osmolaire), injecté à un volume minimum, sans ventriculographie.

\section{Poumons}

Pour connaître les recommandations relatives aux corticostéroïdes et à la protection des poumons, voir la recommandation 5.2.

\section{Pratique actuelle au Canada}

Les mesures suivantes sont des aspects couramment acceptés dans la pratique et sont approuvées a priori :

- Oxymétrie pulsée, gazométries artérielles en série, aspiration par tube endotrachéal, radiographie pulmonaire, bronchoscopie et lavage broncho-alvéolaire.

- Ventilation mécanique axée sur les valeurs cibles suivantes :

- fraction d'oxygène inspiré $\left(\mathrm{FIO}_{2}\right)$ titrée de façon à maintenir une saturation en oxygène de $\geq 95 \%$ et une pression partielle d'oxygène dans le sang artériel $\left(\mathrm{PaO}_{2}\right) \mathrm{de}$ $\geq 80 \mathrm{~mm} \mathrm{Hg}$

- $\mathrm{pH}: 7,35-7,45 ; \mathrm{PaCO}_{2}: 35-45 \mathrm{~mm} \mathrm{Hg}$;

- Pression positive en fin d'expiration (PEEP) : $5 \mathrm{~cm} \mathrm{H}_{2} \mathrm{O}$.

\section{Recommandation 9.1 : Déficit de l’oxygénation}

Lorsque le rapport de la pression partielle d'oxygène dans le sang artériel sur la fraction d'oxygène inspiré (P/F) est de $<300$, nous recommandons ce qui suit :

- Le traitement de routine doit comprendre une rotation en position latérale aux 2 heures.

- Une aspiration et une physiothérapie de routine doivent faire partie des soins courants.

- Une PEEP de $5 \mathrm{~cm} \mathrm{H}_{2} \mathrm{O}$ est recommandée, mais des augmentations périodiques jusqu'à $15 \mathrm{~cm} \mathrm{H}_{2} \mathrm{O}$ constituent une forme acceptable de recrutement alvéolaire.

- Des inspirations soutenues (pression inspiratoire de pointe de $30 \mathrm{~cm} \mathrm{H}_{2} \mathrm{O}$ pendant une période de $30 \mathrm{à} 60 \mathrm{sec}$ ) sont une forme acceptable de recrutement alvéolaire.

- Il faut établir au besoin un traitement diurétique visant à rétablir la normovolémie.

\section{Considérations clés}

- On évalue le rapport $\mathrm{P} / \mathrm{F}$ à partir d'une PEEP de $5 \mathrm{~cm} \mathrm{H}_{2} \mathrm{O}$ et d'une $\mathrm{FIO}_{2}$ de I,o.

- Il faut utiliser périodiquement des manœuvres de recrutement chez tous les donneurs, sans égard au rapport $\mathrm{P} / \mathrm{F}$, et les maintenir durant toute la période peropératoire.

- La position ventrale n'est pas recommandée pour les adultes et les enfants donneurs.

\section{Recommandation 9.2 : Limites inférieures du rapport $P / F$}

- Il ne doit y avoir aucune limite inférieure prédéterminée du rapport $\mathrm{P} / \mathrm{F}$ qui empêche la transplantation.

- Il faut tenir compte du moment de l'évaluation, des variations temporelles, de la réponse au recrutement alvéolaire et de l'état du receveur.

- Dans les cas d'atteinte pulmonaire unilatérale, il est essentiel de déterminer la $\mathrm{PO}_{2}$ dans le sang veineux pulmonaire déterminée pendant la chirurgie pour que l'évaluation de la fonction pulmonaire controlatérale soit fiable.

\section{Considérations clés}

Aucune.

\section{Recommandation 9.3 : Cibles optimales pour le volume courant et la pression inspiratoire de pointe}

Nous recommandons que :

- le volume courant soit de 8 à $\mathrm{ro} \mathrm{mL} / \mathrm{kg}$;

- la limite supérieure de la pression inspiratoire de pointe soit de $\leq 30 \mathrm{~cm} \mathrm{H}_{2} \mathrm{O}$.

\section{Considération clé}

On utilise actuellement des stratégies de protection des poumons chez les patients en détresse respiratoire aiguë, ou qui risquent de l'être, lorsque la ventilation limitée par la pression est définie par une pression respiratoire de pointe de $<35 \mathrm{~cm} \mathrm{H}_{2} \mathrm{O}$ et un volume courant de 6 à $\left.8 \mathrm{~mL} / \mathrm{kg}\right)^{15}$. Les avantages de ces stratégies s'appliquent aux patients en détresse respiratoire aiguë et il n'existe pas de données correspondantes dans le cas des donneurs d'organes.

\section{Recommandation 9.4 : Bronchoscopie et thérapie aux antimicrobiens}

- L'expert du centre hospitalier local peut pratiquer la bronschoscopie et les résultats peuvent être transmis au chirurgien responsable de la transplantation.

- Il faut choisir le traitement aux antimicrobiens en fonction des résultats d'une coloration de Gram, d'une culture 
ou de la présence présumée ou confirmée d'une bronchopneumonie.

- Un traitement empirique aux antibiotiques à spectre large n'est pas indiqué systématiquement, mais on peut l'administrer aux donneurs exposés à un risque élevé de bronchopneumonie.

- La durée du séjour aux soins intensifs ne constitue pas une indication indépendante pour une thérapie aux antimicrobiens.

\section{Considérations clés}

- Il faudrait mettre au point une technologie qui permettra :

- d'examiner à distance les images de bronchoscopie et de radiographie pulmonaire;

- d'établir une communication trilatérale entre les services des soins intensifs, les organisations de prélèvement d'organes et les chirurgiens responsables de la transplantation.

- Il faut éviter les antimicrobiens néphrotoxiques, dans la mesure du possible.

\section{Foie}

\section{Pratique actuelle au Canada}

Les mesures suivantes sont des aspects couramment acceptés dans la pratique et sont approuvées a priori :

- On évalue les aspects suivants des donneurs possibles d'un foie :

- antécédents d'ictère, d'hépatite ou de consommation excessive d'alcool;

- mesure des taux d'aspartate aminotransférase (AST), d'alanine aminotransférase (ALT) et de bilirubine (directe et indirecte, si possible), ainsi que du ratio international normalisé (RI) ou du temps de prothrombine [TP], aux $6 \mathrm{~h}$;

- taux sériques d'électrolytes, de créatinine et d'urée; présence de l'antigène de surface de l'hépatite $B(\mathrm{AgHBs})$

l'anticorps du virus de l'hépatite B (Ac anti VHB) et de l'anticorps du virus de l'hépatite C (Ac anti VHC).

\section{Recommandation 10.1 : Limites supérieures des concentrations d'aspartate aminotransférase (AST) et d'alanine aminotransférase (ALT) hépatiques}

Nous recommandons qu'il n'y ait aucune limite supérieure des concentrations d'AST et d'ALT hépatiques qui empêche de transplanter un foie. Il faut offrir tous les foies. Les décisions relatives à la transplantabilité dépendent de l'état de l'organe, des tendances de la fonction hépatique au fil du temps et de l'état du receveur.

\section{Considérations clés}

Aucune.

\section{Recommandation 10.2 : Échographie du foie}

Nous recommandons que rien n'oblige les donneurs de foie éventuels à subir une échographie du foie.

\section{Considérations clés}

Aucune.

\section{Recommandation 10.3 : Indications pour une biopsie du foie}

Nous recommandons qu'une biopsie percutanée du foie, guidée par échographie, soit indiquée dans les cas suivants et qu'on l'effectue à l'unité des soins intensifs avant le prélèvement, en collaboration avec l'équipe chargée de la transplantation du foie, pour permettre la prise de décisions sur la transplantabilité :

- poids $>$ Ioo $\mathrm{kg}$ ou indice de masse corporelle $>30$ ou présence d'anticorps du VHC chez le donneur et

- prélèvement différé, c'est-à-dire lorsqu'aucune équipe chirurgicale ne peut procéder immédiatement au prélèvement du foie.

Une biopsie peropératoire, pratiquée par l'équipe chargée du prélèvement, est recommandée dans tous les autres cas où une biopsie du foie est indiquée.

Si une biopsie est indiquée mais ne peut être pratiquée aux soins intensifs, il faut offrir le foie et laisser l'équipe chargée de la transplantation en déterminer la transplantabilité.

\section{Considérations clés}

Aucune.

\section{Rein}

\section{Pratique actuelle au Canada}

Les mesures suivantes sont couramment acceptées dans la pratique et sont approuvées a priori :

- Le seuil de fonction optimale pour une transplantation est fixé à une clairance de la créatinine normale ( $>80 \mathrm{~mL} / \mathrm{min} / \mathrm{I}, 73 \mathrm{~m}^{2}$ ). Une mesure du taux de créatinine sérique ou un calcul de la clairance de la créatinine qui donne un résultat anormal ne signifie toutefois pas nécessairement que les reins d'un donneur ne peuvent être transplantés.

- Une analyse d'urine est essentielle pour exclure toute anomalie rénale.

- Il faut mesurer aux 6 heures les concentrations de créatinine et d'urée sérique (azote uréique du sang).

\section{Recommandation 11.1 : Clairance de la créatinine}

Nous recommandons de mesurer la clairance de la créatinine au moyen de l'équation de Cockroft-Gault. Il n'est pas indiqué de recueillir des échantillons d'urine pour déterminer la clairance de la créatinine.

\section{Considération clé}

Il n'existe aucune contre-indication absolue à un don de rein fondée sur la concentration de créatinine sérique ou la clairance de la créatinine seulement.

\section{Recommandation 11.2 : Échographie rénale}

Nous recommandons de procéder à une échographie rénale 
au cas par cas, en tenant compte de facteurs tels que des antécédents de néphropathie.

\section{Considération clé}

En général, il n'existe aucune indication formelle pour une échographie rénale, car cet examen a tendance à produire peu d'information.

\section{Recommandation 11.3 : Indications pour une biopsie du rein}

Nous recommandons de tenir compte des variables ci-dessous pour déterminer s'il faut procéder à une biopsie peropératoire du rein au moment du prélèvement afin de décider s'il est transplantable.

- Sujet âgé de plus de 65 ans ou plus jeune qui présente n'importe lequel des antécédents suivants :

- concentration de créatinine > $133 \mu \mathrm{mol} / \mathrm{L}$;

- hypertension;

- diabète;

- analyse d'urine qui donne des résultats anormaux.

\section{Considération clé}

Il faut procéder à une évaluation histologique et déterminer s'il y a glomérulosclérose ou vasculopathie, ou les deux, avant d'exclure les reins. Il faut pratiquer la biopsie à la salle d'opération, au moment du prélèvement, plutôt qu'aux soins intensifs.

\section{Autres défis systémiques}

\section{Moment optimal pour le prélèvement de s organes et la prise des décisions sur la transplantabilité}

\section{Recommandation 12.1 : Moment optimal pour le prélèvement des organes}

Il est important de prendre le temps nécessaire aux soins intensifs pour optimiser la fonction de plusieurs organes afin d'améliorer les résultats des transplantations. Une réanimation et une réévaluation peuvent atténuer une dysfonction réversible des organes. Cette période de traitement peut durer de 12 à 24 heures et il faut y conjuguer de fréquentes réévaluations afin de démontrer que la fonction des organes évolue vers les cibles définies.

Lorsque la fonction des organes est optimisée, il faut les prélever d'urgence.

\section{Pratique actuelle au Canada}

En général, après qu'on a déclaré le décès neurologique et obtenu le consentement nécessaire à un don d'organes, le centre hospitalier s'efforce d'organiser les aspects logistiques du don et de procéder au prélèvement le plus rapidement possible.

\section{Considérations clés}

- Il faut modifier le paradigme actuel des soins en fonction des situations suivantes, qu'il est possible de corriger ou d'améliorer par une réanimation et une réévaluation :

- dysfonction myocardique ou cardiovasculaire;

- déficit de l'oxygénation lié à une atteinte pulmonaire qui pourrait être réversible;

- infections bactériennes invasives;

- hypernatrémie;

- tendances temporelles des concentrations d'AST et d'ALT ;

- tendances temporelles des concentrations de créatinine;

- toute autre situation qui pourrait être traitable.

- Il faut tenir compte, dans les discussions sur le consentement au don, de la prolongation de la période de soins prodigués au donneur aux soins intensifs afin d'optimiser l'issue de la transplantation et elle doit respecter les volontés de la famille ou du fondé de pouvoir.

\section{Recommandation 12.2 : Décisions relatives à la transplantabilité}

Nous recommandons qu'il n'y ait aucun facteur démographique ni seuil de dysfonction prédéfini qui empêche d'obtenir le consentement pour le don d'un seul organe ou d'offrir des organes aux fins de transplantation et que :

- l'on demande le consentement pour tous les organes;

- l'on offre tous les organes dans le contexte des cadres juridique et réglementaire existants;

- les décisions finales quant à la transplantabilité incombent à chaque programme de transplantation représenté par les médecins responsables de la greffe de chaque type d'organe.

\section{Pratique actuelle au Canada}

Il existe des variations importantes dans la pratique.

\section{Considérations clés}

- Les services de prélèvement et de transplantation doivent rendre compte de la non-utilisation d'organes. Les données limitées fournies au Registre canadien du remplacement d'organes sur les raisons de la non-utilisation d'organes sont insuffisantes.

- Il faut établir un lien entre l'utilisation des organes et la transplantation du greffon correspondant et les résultats chez les patients.

- Il faut aborder les questions relatives aux virus ou aux cellules cancéreuses transmissibles conformément aux normes et aux lignes directrices en vigueur au Canada ${ }^{16}$.

\section{Adaptations en fonction de l'âge chez les enfants}

\section{Recommandation 13 : Adaptation en fonction de l'âge chez les enfants}

Nous recommandons que les recommandations I à I2 s'appliquent aux nourrissons, aux enfants et aux adolescents avec les réserves suivantes. (Les chiffres correspondent aux recommandations chez les adultes.) 


\section{Critères principaux}

- Le donneur d'organe en pédiatrie :

- a de l'âge de la naissance à 18 ans;

- est traité dans une unité de soins intensifs en pédiatrie.

- Les recommandations posologiques s'appliquent aux enfants de $\leq 60 \mathrm{~kg}$. Lorsqu'ils pèsent plus de $60 \mathrm{~kg}$, il faut appliquer les doses adultes.

\section{1 : Hypertension artérielle générale liée à la pression} intracrânienne

- Les seuils pour traiter l'hypertension artérielle après le DDN sont les suivants :
Nouveau-nés à 3 mois
$>3$ à 12 mois
I à 12 ans
$>$ I2 à I8 ans

$$
\begin{aligned}
& >90 / 60 \mathrm{~mm} \mathrm{Hg} \\
& >\mathrm{IIO} / 70 \mathrm{~mm} \mathrm{Hg} \\
& >130 / 80 \mathrm{~mm} \mathrm{Hg} \\
& >\mathrm{I} 40 / 90 \mathrm{~mm} \mathrm{Hg}
\end{aligned}
$$

2 : Performance cardiovasculaire, surveillance et soutien hémodynamique

- Des praticiens experts des soins intensifs pédiatriques doivent adapter les traitements en fonction de valeurs cibles générales, plutôt que spécifiques, liées à l'âge. Un guide sur les plages normales de la fréquence cardiaque et de la tension artérielle selon l'âge figure à la rubrique Considérations clés ci-dessous.

\section{1 : Surveillance de la saturation en oxygène du sang} veineux mêlé ou central

- L'oxymétrie du sang veineux central sert actuellement dans de nombreuses unités de soins intensifs pédiatriques au Canada comme méthode de surveillance de patients qui présentent une instabilité hémodynamique et est recommandée chez les enfants donneurs. Il faut adapter le traitement de façon à produire une saturation en oxygène du sang veineux central de $\geq 60 \%$.

\section{3: Indications pour la CAP}

- L'utilisation de la CAP est limitée dans la pratique des soins intensifs en pédiatrie et n'est pas recommandée de routine chez les enfants donneurs. La décision de recourir à une CAP est laissée à la discrétion du praticien de l'unité des soins intensifs pédiatriques qui en maîtrise l'application et l'interprétation.

- La prise d'échocardiographies en série est la méthode préconisée pour réévaluer la fonction myocardique aux fins de la transplantation. Il faut adapter à chaque cas l'utilisation de cette méthode comme outil pouvant guider le traitement hémodynamique.

\section{4 : Agents de première intention pour le soutien} hémodynamique - vasopressine

- La dose de vasopressine recommandée chez les enfants est de 0,0003 à $0,0007 \mathrm{U} / \mathrm{kg} / \mathrm{min}$ (de 0,3 à $0,7 \mathrm{mU} / \mathrm{kg} / \mathrm{min}$ ), jusqu'à concurrence de $2,4 \mathrm{U} / \mathrm{h}$.

\section{5 : Agents de deuxième intention pour le soutien hémodynamique - norépinéphrine, épinéphrine et phényléphrine}

- En l'absence de données tirées de la CAP, il faut adapter le traitement hémodynamique en fonction des évaluations cliniques et biochimiques.

\section{1 : Diabète insipide}

- La dose de vasopressine recommandée chez les enfants est de 0,0003 à $0,0007 \mathrm{U} / \mathrm{kg} / \mathrm{min}$ (de 0,3 à $0,7 \mathrm{mU} / \mathrm{kg} / \mathrm{min}$ ), jusqu'à concurrence de $2,4 \mathrm{U} / \mathrm{h}$.

\section{1 : Hormone thyroïdienne, vasopressine et méthylprednisolone}

- La dose de vasopressine recommandée chez les enfants est de 0,0003 à $0,0007 \mathrm{U} / \mathrm{kg} / \mathrm{min}$ (de 0,3 à $0,7 \mathrm{mU} / \mathrm{kg} / \mathrm{min}$ ), jusqu'à concurrence de $2,4 \mathrm{U} / \mathrm{h}$.

- T4 par voie intraveineuse : La plage posologique précise pour les perfusions de $\mathrm{T}_{4}$ par voie IV n'est pas connue, et sa stabilité en solution et son adhérence possible à la tubulure de plastique en raison de sa nature hydrophobe peuvent modifier son effet biologique. Chez les adultes, les praticiens ont administré à des donneurs éventuels des doses de jusqu'à 300 à $500 \mu \mathrm{g}$, en bol IV. Il s'agit de la plage posologique standard dans les cas de coma myxœdémateux. Étant donné l'étendue de la plage posologique mentionnée dans les publications dans le cas de la $T_{4} \mathrm{IV}$ et le faible risque de toxicité pour cette indication courante, on recommande aussi pour les enfants ${ }^{17}$ la plage des adultes - bol de $20 \mu \mathrm{g}$ par voie IV suivi d'une perfusion de Io $\mu \mathrm{g} / \mathrm{h}$ par voie IV. Une autre possibilité consiste à administrer la $\mathrm{T}_{4}$ par voie intraveineuse, comme suit : bol de Ioo $\mu \mathrm{g}$ IV, suivi d'une perfusion IV de 25 à 50 $\mu \mathrm{g}$ aux $\mathrm{I} 2$ heures.

\section{1 : Évaluation initiale de la fonction cardiaque}

- Une série d'échocardiographies constitue la méthode recommandée pour évaluer la fonction myocardique aux fins de la transplantation. Il faut procéder à l'échocardiographie initiale seulement après que le sujet a été stabilisé par une réanimation volémique adéquate.

- Il faut répéter l'échocardiographie aux 6 à 12 heures dans les conditions suivantes :

- l'échocardiographie bidimensionnelle initiale révèle une fraction d'éjection de $\leq 40 \%$, ou

- intensification des mesures de soutien, définie par une dose de dopamine de > $\mathrm{ro} \mu \mathrm{g} / \mathrm{kg} / \mathrm{min}$, l'emploi d'agents vasopresseurs, ou les deux.

\section{1 : Clairance de la créatinine}

- Chez les enfants de plus d'un an, un taux normal de clairance de la créatinine est de $>80 \mathrm{~mL} / \mathrm{min} / \mathrm{I}, 73 \mathrm{~m} 2$, selon l'estimation basée sur la formule de Schwartz ${ }^{18}$.

11.3 : Indications pour une biopsie du rein

- Taux de créatinine supérieur à la normale pour l'âge.

\section{Considérations clés}

Voir Tableau I. 
Tableau 1 : Plages normales de la fréquence cardiaque et de la tension artérielle selon l'âge.

\begin{tabular}{lccc}
\hline & $\begin{array}{l}\text { Fréquence } \\
\text { cardiaque; } \\
\text { battements/ } \\
\text { minute }\end{array}$ & $\begin{array}{l}\text { TA systolique } \\
(\mathrm{mm} \mathrm{Hg})\end{array}$ & $\begin{array}{l}\text { TA diastolique } \\
(\mathrm{mm} \mathrm{Hg})\end{array}$ \\
\hline Âge & $100-150$ & $65-85$ & $45-55$ \\
\hline 0 à 3 mois & $90-120$ & $70-90$ & $50-65$ \\
\hline 3 à 6 mois & $80-120$ & $80-100$ & $55-65$ \\
6 à 12 mois & $70-110$ & $90-105$ & $55-70$ \\
1 à 3 ans & $65-110$ & $95-110$ & $60-75$ \\
3 à 6 ans & $60-95$ & $100-120$ & $60-75$ \\
\hline 6 à 12 ans & $55-85$ & $110-135$ & $65-85$ \\
\hline$>12$ ans & & & \\
\hline
\end{tabular}

Note : TA $=$ tension artérielle

Adapté de : Mathers LH, Frankel LR. Stabilization of the Critically III Child.

Nelson textbook of pediatrics, $17^{\mathrm{e}}$ édition, $2004^{19}$.

Cet article a été critiqué par des pairs.

Unité de soins intensifs pédiatriques, Hôpital de Montréal pour enfants, Centre universitaire de santé McGill, Montréal (Qué.) (Shemie); Programme de transplantation cardiaque, Hôpital Toronto General, Réseau de santé universitaire (Ross); Programme de transplantations d'organes de l'appareil digestif, Hôpital Toronto General, Réseau de santé universitaire (Greig); Chirurgie générale, Programme de dons d'organes et de tissus, Hôpital d'Ottawa (Pagliarello); Unité de soins intensifs, Traumatologie et neurochirurgie, Hôpital St. Michael's, Université de Toronto, Toronto (Ont.) (Baker); Soins intensifs aux adultes, Hôpital Foothills, Calgary (Alb.) (Doig); Réseau Trillium pour le don de vie (Baker); Société canadienne de soins intensifs (Shemie, Pagliarello, Baker, Doig, Guest); Société canadienne des anesthésiologistes (Baker); Registre canadien du remplacement d'organes (Greig); Société canadienne de transplantation (Greig, Ross, Cockfield, Keshavjee, Rao, Nickerson); Association canadienne de transplantation (Brand, Young); Programme de transplantation du rein, Hôpital de l'Université de l'Alberta, Edmonton (Alb.) (Cockfield); Programme de transplantation du poumon de Toronto, Réseau de santé universitaire, Université de Toronto, Toronto (Ont.) (Keshavjee); Laboratoire d'immunogénétique, Centre des services de santé de l'Université du Manitoba, Winnipeg (Man.) (Nickerson); Programme de transplantation cardiaque, Réseau de santé universitaire, Université de Toronto, Toronto (Ont.) (Rao); Département de médecine des soins intensifs, Hôpital Sunnybrooke and Women's College, Toronto (Ont.) (Guest); Conseil canadien pour le don et la transplantation (Shemie, Young, Doig); Programme de transplantation de la Saskatchewan (Brand).

Voir à l'Annexe 4 la liste complète des membres des comités.

Intérêts concurrents: Aucun déclaré pour Sam Shemie, Heather Ross, Joe Pagliarello, Andrew Baker, Tracy Brand, Sandra Cockfield, Shaf Keshavjee, Peter Nickerson, Vivek Rao, Cameron Guest, Kimberly Young ou Christopher Doig. Paul Greig a reçu des honoraires de conférencier et des subventions de voyage de Hoffmann La-Roche et Fujisawa Canada Inc. pout assister aux réunions.

Remerciements : Ces recommandations ont reçu l'aval de la Société canadienne de soins intensifs, de l'Association canadienne de transplantation, de la Société canadienne de transplantation et du Conseil canadien pour le don et la transplantation.

Nous reconnaissons Le Conseil canadien pour le don et la transplantation a fourni de l'aide financière pour préparer et organiser le forum; la Société canadienne de soins intensifs, l'Association canadienne de transplantation et la Société canadienne de transplantation ont collaboré à la tenue du forum. et l'aide financière obtenue du Conseil canadien pour le don et la transplantation et le processus de consultation pris en charge par StrachanTomlinson.

\section{RÉFÉRENCES}

I. Hornby K, Shemie SD. Donor organ management : survey of guidelines and eligibility criteria. Edmonton: Conseil canadien pour le don et la transplantation; 2004.

2. Hornby K, Ross H, Keshavjee S, et al. Factors contributing to non-utilization of heart and lungs after consent for donation: a Canadian multicentre study. Can J Anaesth 2006; sous presse.

3. Tung P, Kopelnik A, Banki N, et al. Predictors of neurocardiogenic injury after subarachnoid hemorrhage. Stroke 2004; 35(2): 548-5I.

4. Novitzky D. Detrimental effects of brain death on the potential organ donor. Transplant Proc 1997; 29: 3770-2.

5. Shivalkar B, Van Loon J, Wieland W, et al. Variable effects of explosive or gradual increase of intracranial pressure on myocardial structure and function. Circulation I993; 87(I):230-9.

6. D'Amico TA, Meyers CH, Koutlas TC, et al. Desensitization of myocardial betaadrenergic receptors and deterioration of left ventricular function after brain death. $J$ Thorac Cardiovasc Surg I995; IIo(3): 746-5I.

7. Wheeldon DR, Potter CD, Oduro A, et al. Transforming the "unacceptable" donor: outcomes from the adoption of a standardized donor management technique. $J$ Heart Lung Transplant I995; I4(4): 734-42.

8. Richardson DW, Robinson AG. Desmopressin. Ann Intern Med I985; 103 (2): 228-39.

9. Totsuka E, Dodson F, Urakami A, et al. Influence of high donor serum sodium levels on early postoperative graft function in human liver transplantation: effect of correction of donor hypernatremia. Liver Transpl Surg I999; 5(5): 42I-8.

Io. Rosendale JD, Kauffman HM, McBride MA, et al. Hormonal resuscitation yields more transplanted hearts, with improved early function. Transplantation 2004; 78(2) suppl I: 17.

II. Follette DM, Rudich SM, Babcock WD. Improved oxygenation and increased lung donor recovery with high-dose steroid administration after brain death. J Heart Lung Transplant I998; I7(4): 423-9.

I2. Hunt SA, Baldwin J, Baumgartner W, et al. Cardiovascular management of a potential heart donor: a statement from the Transplantation Committee of the American College of Cardiology. Crit Care Med i996; 24(9): 1599-6or.

I3. McGiffin DC, Savunen T, Kirklin JK, et al. A multivariable analysis of pretransplantation risk factors for disease development and morbid events. J Thorac Cardiovasc Surg I995; I09(6): I08I-9.

I4. Zaroff JG, Rosengard BR, Armstrong WF, et al. Consensus conference report maximizing use of organs recovered from the cadaver donor: cardiac recommendations. Circulation 2002; 106(7): 836-4I.

15. Ventilation with lower tidal volumes as compared with traditional tidal volumes for acute lung injury and the acute respiratory distress syndrome. The Acute Respiratory Distress Syndrome Network. N Engl J Med 2000; 342(I8): I30I-8.

I6. Cellules, tissus et organes destinés à la transplantation et à la reproduction assistée exigences générales (Z900.I-03). Mississauga (Ont.) : Association canadienne de normalisation; 2003

17. Rodriguez I, Fluiters E, Perez-Mendez LF, et al. Factors associated with mortality of patients with myxoedema coma: prospective study in II cases treated in a single institution. JEndocrinol 2004; I8o(2): 347-50.

I8. Schwartz GJ, Brion LP, Spitzer A. The use of plasma creatinine concentration for estimating glomerular filtration rate in infants, children, and adolescents. Pediatr Clin North Am I987; 34(3): 571-90.

I9. Mathers LH, Frankel LR. Stabilization of the critically ill child. Dans Behrman RE,Kliegman RM, Jenson HB (dir.). Nelson Textbook of Pediatrics ( $17^{e}$ édition). Philadelphie (Penn.) : Saunders; 2004.

20. À propos de l'application des connaissances. Ottawa (Ont.) : Instituts de recherche en santé du Canada; 2005. Disponible à : www.cihr-irsc.gc.ca/f/29418.html (consulté le $\mathrm{I}^{\text {er }}$ déc. 2005).

Correspondance : $D^{r}$ Sam D. Shemie, Unité de soins intensifs pédiatriques, Hôpital de Montréal pour enfants, Centre universitaire de santé McGill, Montréal (Québec) $\mathrm{H}_{3} \mathrm{H}_{1} P_{3}$; sam.shemie@muhc.mcgill.ca

Demandes de réimpressions : $M^{\text {me }}$ Kimberly Young, Conseil canadien pour le don et la transplantation, I702-8215 II2 St., Edmonton (Alberta) T6G 2C8 (téléphone : 7804095652 ; courriel : kimberly.young@ccdt.ca) 


\section{Annexe 1 : Programme national de recherche}

Les participants ont conclu que les recherches cliniques en cours susceptibles d'étayer les recommandations issues du Forum comportent des limites importantes. Ils ont encouragé la mise sur pied d'initiatives de recherche locales et multicentriques en plus des projets menés à l'échelle nationale et internationale. Les sujets de recherche possibles qui suivent sont issus des ateliers.

Sujets qui se prêtent aux méthodes fondées sur les enquêtes

- Enquête transversale sur les pratiques actuelles de soutien cardiorespiratoire chez les donneurs, y compris les valeurs cibles et les traitements hémodynamiques, la ventilation et les stratégies de recrutement pulmonaire.

\section{Sujets qui se prêtent à des études d'observation}

Il est possible d'approfondir les questions suivantes par des enquêtes, des études prospectives et rétrospectives et des analyses de bases de données :

- Rôle d'une série d'échocardiographies ou d'une échocardiographie d'effort sensibilisée par la dobutamine dans l'évaluation des patients qui ont une fonction myocardique réduite, par rapport au CAP seul.

- Effet d'une exposition à un agent de contraste radiologique (durant l'angiographie cérébrale ou la coronarographie) sur la fonction du greffon rénal.

- Étude des facteurs qui contribuent à la variabilité des taux d'utilisation des organes entre les centres et mise en corrélation avec la fonction des organes après la transplantation.

- Combinaisons optimales d'agents vasopresseurs et inotropes et effet d'un prélèvement différé sur l'utilisation des organes.

Sujets qui se prêtent à des études interventionnelles non randomisées

- Effet d'une série de manœuvres de recrutement des poumons chez les donneurs d'organes sur le rapport $\mathrm{PaO}_{2} / \mathrm{FIO}_{2}$ et sur les taux de prélèvement des poumons.

- Études pharmacologiques sur des doses de $T_{4}$ et de $T_{3}$ administrées par voie orale et intraveineuse chez des êtres humains, notamment sur la cinétique, les effets biologiques, la posologie optimale, les temps de conversion dans les tissus périphériques et l'effet des corticostéroïdes.

Sujets qui se prêtent à des essais cliniques contrôlés randomisés

- Une hormonothérapie combinée améliore-t-elle les paramètres hémodynamiques, la fonction des organes ou leur utilisation?

- Un CAP et un traitement axé sur des valeurs cibles améliorent-ils les paramètres hémodynamiques, la fonction des organes ou leur utilisation?

- Évaluation d'une prophylaxie par la $\mathrm{N}$-acétylcystéine pour prévenir une néphropathie induite par des agents de contraste et une reprise retardée de la fonction des reins provenant de donneurs décédés. 


\section{Annexe 2 : Logistique et application des connaissances}

Pour optimiser la prise en charge des donneurs et l'utilisation des organes aux fins de transplantation, il faut diffuser à grande échelle les recommandations issues du Forum et mobiliser aussi un grand nombre de personnes et d'organisations de tous les secteurs du système de santé. Pour répondre à ces exigences interdépendantes, un groupe de la logistique et de l'application des connaissances (LAC) s'est réuni tout au long du Forum pour discuter de la question suivante :

Comment assurer que les consensus dégagés au cours du Forum seront transposés de façon efficiente et efficace dans la pratique afin d'améliorer le plus tôt possible la prise en charge médicale?

Les membres du groupe LAC ont formulé des recommandations qui aideront à résoudre les problèmes de logistique et d'application des connaissances sur les plans de la pratique clinique et des changements généraux.

\section{Défis logistiques}

Le groupe LAC a discuté de façons de faciliter la mise en œuvre des lignes directrices (p. ex., une série normalisée de lignes directrices favoriserait l'excellence clinique et entraînerait l'élaboration de politiques hospitalières constructives) et des facteurs qui nuiraient à leur application (p. ex., répercussions sur les ressources de séjours prolongés aux soins intensifs, réduction des possibilités de récupération d'organes à cause du manque d'accès aux salles d'opération et absence de définition de ce qui constitue un hôpital de prélèvement d'organes). Il a formulé les recommandations suivantes pour s'attaquer aux problèmes logistiques qui pourraient nuire à l'application des lignes directrices:

- Produire une analyse des coûts et avantages des dons, y compris des incidences pour le système de soins de santé et des questions liées à la qualité de vie.

- Surveiller la résolution dans chaque cas des problèmes logistiques et leurs répercussions sur l'utilisation optimale des organes, et en faire rapport.

\section{Défis liés à l'application des connaissances}

Les Instituts de recherche en santé du Canada (IRSC) décrivent l'application des connaissances comme le processus qui permet de transférer les résultats de recherche des producteurs de connaissances aux utilisateurs de ces connaissances pour le bénéfice de tous les Canadiens ${ }^{20}$. Il comporte trois éléments interdépendants :

- l'échange des connaissances;

- la synthèse des connaissances;

- l'application éthique du savoir.

L'application des connaissances vise à améliorer les processus, les services et les produits de santé, ainsi que le système de santé même.

Les membres du groupe LAC ont formulé les recommandations suivantes pour maximiser l'application des connaissances et accroître l'utilisation des organes :

- Élaborer des politiques d'orientation du processus de don qui définissent clairement les rôles et les responsabilités des professionnels de la santé, par ex. : intégration des recommandations du forum aux protocoles opératoires normalisés des organismes de prélèvement d'organes et des centres hospitaliers.

- Encourager l'échange d'information à l'échelle nationale et internationale pour améliorer les connaissances et la qualité des soins au Canada et dans d'autres pays.

- Voir à ce qu'aucun problème de distribution n'entrave la distribution des organes et empêche ainsi de les utiliser.

- Considérer l'identification des donneurs possibles d'organes et de tissus comme un élément qui fait partie intégrante des soins de qualité offerts en fin de vie pour maximiser l'utilisation des organes.

- Offrir aux professionnels de la santé une formation continue et des occasions d'améliorer leurs compétences pour appuyer la prestation de soins de qualité aux donneurs et aux membres de leur famille. Créer des outils rapides à consulter sur les protocoles et les guides.

- Appuyer la mise en œuvre des recommandations du Forum dans les organismes et les établissements affiliés au Forum.

- Appuyer des initiatives qui visent à accroître la production de rapports et l'obligation de rendre compte. Encourager la normalisation des données et de la terminologie aux échelons tant national qu'international. Concevoir des outils de mesure de grande qualité qui se répercutent directement sur le processus de don, y compris l'identification des donneurs, les demandes de consentement, l'octroi du consentement et l'utilisation des organes. 


\section{Annexe 3 : Règlement sur la prise en charge de donneurs d'organes}

Il est important de prendre le temps nécessaire, aux soins intensifs, pour optimiser la fonction de multiples organes afin d'améliorer l'issue des transplantations. Une réanimation et une réévaluation permettent d'améliorer une dysfonction réversible des organes (dysfonction myocardique et cardiovasculaire, déficit de l'oxygénation lié à une atteinte pulmonaire qui pourrait être réversible, infections bactériennes invasives, hypernatrémie) et de suivre les tendances temporelles des taux d'aspartate aminotransférase (AST), d'alanine aminotransférase (ALT) et de créatinine ou de tout autre état pouvant être traitable. Cette période de traitement peut durer de 12 à 24 heures et il faut y ajouter de fréquentes réévaluations montrant que la fonction des organes s'améliore et évolue vers les cibles définies. Lorsque la fonction des organes est optimisée, il faut les prélever d'urgence. Il n'existe aucun facteur démographique ni seuil de dysfonction prédéfini qui empêche de consentir à un don et d'offrir des organes aux fins de transplantation.

\begin{tabular}{lll}
\hline Donneurs adultes & \\
\hline Surveillance Sonde urinaire pour assurer le drainage, contrôle rigoureux des ingérés et des excrétés.
\end{tabular}

standard $\quad$ Sonde nasogastrique pour assurer le drainage.

- Prise des signes vitaux aux heures.

- Oxymétrie pulsée, électrocardiographie à 3 dérivations.

- Pression veineuse centrale.

- Surveillance de la tension artérielle par cathéter artériel.

- Utilisation facultative d'un CAP.

$\begin{array}{ll}\text { Analyses de } & \text { - Gazométrie du sang artériel, électrolytes, glycémie aux } 4 \mathrm{~h} \text { et au besoin. } \\ \text { laboratoire } & \text { - Formule sanguine aux } 8 \mathrm{~h} \text {. }\end{array}$

- Azote uréique du sang et créatinine aux $6 \mathrm{~h}$.

- Analyse d'urine.

- AST, ALT, bilirubine (totale et directe), RIN (ou temps de Quick) et temps de céphaline aux $6 \mathrm{~h}$.

Surveillance et traitement hémodynamiques

Objectifs généraux : fréquence cardiaque entre 60 et 120 battements/minute, tension artérielle systolique $\geq 70 \mathrm{~mm} \mathrm{Hg}$.

- Réanimation liquidienne pour maintenir une normovolémie, pression veineuse centrale de 6 à $10 \mathrm{~mm} \mathrm{Hg}$.

- Si la tension artérielle est $\geq 160 / 90 \mathrm{~mm} \mathrm{Hg}$, il faut alors : procéder au sevrage des agents inotropes et vasopresseurs, et commencer au besoin à administrer

- le nitroprussiate, à une dose de 0,5 à $5,0 \mu \mathrm{g} / \mathrm{kg} / \mathrm{min}$, ou

- l'esmolol, à une dose de 100 à $500 \mu \mathrm{g} / \mathrm{kg}$ en bol, puis à une dose de 100 à $300 \mu \mathrm{g} / \mathrm{kg} / \mathrm{min}$.

- Lactate sérique aux 2 à $4 \mathrm{~h}$.

- Oxymétrie du sang veineux mêlé aux 2 à $4 \mathrm{~h}$; titrer le traitement pour produire une $\mathrm{MVO}_{2} \geq 60 \%$.

Agents indiqués comme traitement de soutien hémodynamique

Indications pour le CAP

- Dopamine : $\leq 10 \mu \mathrm{g} / \mathrm{kg} / \mathrm{min}$.

- Vasopressine : $\leq 2,4 \mathrm{U} / \mathrm{h}(0,04 \mathrm{U} /$ minute $)$.

- Norépinéphrine, épinéphrine et phényléphrine (la prudence s'impose avec des doses de plus de $0,2 \mu \mathrm{gg} / \mathrm{kg} / \mathrm{min}$ ).

- Fraction d'éjection $\leq 40 \%$ déterminée par échocardiographie bidimensionnelle, et (ou)

- Dopamine $>10 \mu \mathrm{g} / \mathrm{kg} / \mathrm{min}$ (ou l'équivalent), et (ou)

- Traitement vasopresseur de soutien (excluant la vasopressine si cette dernière fait partie d'une hormonothérapie), et (ou)

- Intensification des mesures de soutien.

\section{Glycémie et nutrition}

- Perfusions routinières de dextrose par voie intraveineuse

- Mise en place ou maintien d'une alimentation entérale, selon la tolérance.

- Maintien d'une alimentation parentérale déjà en place.

- Instauration et réglage d'une insulinothérapie par perfusion pour maintenir une glycémie de 4 à $8 \mathrm{mmol} /$.

Liquides et
électrolytes

- Débit urinaire de 0,5 à $3 \mathrm{~mL} / \mathrm{kg} / \mathrm{h}$.

- Natrémie 130 à $150 \mathrm{mM}$.

- Taux de potassium, de calcium, de magnésium et de phosphate dans les plages normales.

Diabète insipide Caractéristiques:

- Débit urinaire $>4 \mathrm{~mL} / \mathrm{kg} / \mathrm{h}$ associé à

- Une élévation du taux de Na sérique $\geq 145 \mathrm{mmol} / \mathrm{l}$ et (ou)

- Une élévation de l'osmolarité sérique $\geq 300 \operatorname{mos} M$ et (ou)

- Une réduction de l'osmolarité urinaire $\leq 200 \operatorname{mos} M$.

Traitement (à titrer de façon à produire un débit urinaire de $\leq 3 \mathrm{~mL} / \mathrm{kg} / \mathrm{h}$ ) :

- Perfusion de vasopressine par voie intraveineuse (IV) à $\leq 2,4 \mathrm{U} / \mathrm{h}$, et (ou)

- DDAVP intermittente, 1-4 $\mu \mathrm{g}$ IV, ensuite 1-2 $\mu \mathrm{g}$ IV aux $6 \mathrm{~h}$ (il n'y a pas vraiment de limite supérieure de dose; il faut la titrer en fonction du débit urinaire visé).

\section{Hormonothérapie Caractéristiques :}

combinée

- Tétra-ïodothyronine (T4) - bol de $20 \mu \mathrm{g} \mathrm{IV}$, suivi d'une perfusion IV de $10 \mu \mathrm{g} / \mathrm{h}$ (ou bol de $100 \mu \mathrm{g}$ IV, suivi d'une perfusion IV de $50 \mu$ gux 12 heures)

- Vasopressine - bol de $1 \mathrm{U}$ IV, suivi d'une perfusion IV de 2,4 U/h.

- Méthylprednisolone $-15 \mathrm{mg} / \mathrm{kg}(\leq 1 \mathrm{~g}) \mathrm{IV}$, aux $24 \mathrm{~h}$.

\section{Indications :}

- Fraction d'éjection $\leq 40 \%$ indiquée par échocardiographie bidimensionnelle, ou

- Instabilité hémodynamique (dont état de choc ne répondant pas au rétablissement d'une normovolémie et nécessitant un traitement vasoactif de soutien [dopamine à une dose $>10 \mu \mathrm{g} / \mathrm{min}$ ou tout agent vasopresseur]).

- Il faut envisager de l'utiliser chez tous les donneurs. 
Hématologie - Hémoglobine optimale : 90-100 g/L; pour les donneurs instables, le niveau le plus bas acceptable est de $70 \mathrm{~g} / \mathrm{L}$.

- Plaquettes, RIN, temps de céphaline : aucune valeur cible prédéterminée, transfusions dans les cas d'hémorragies

cliniquement importantes.

- Aucune exigence particulière ne s'applique aux transfusions.

Microbiologie (de référence, quotidienne et au besoin)

Ordonnances particulières au cœur

- Hémocultures quotidiennes.

- Cultures d'urine quotidiennes.

- Cultures quotidiennes d'échantillons prélevés par tube endotrachéal.

- Antibiothérapie dans les cas d'infection présumée ou confirmée.

- Électrocardiogramme à 12 dérivations.

- Mesure du taux de troponine I ou T, aux 12 h.

- Échographie bidimensionnelle :

- Seulement après une réanimation liquidienne et hémodynamique;

- Si la fraction d'éjection $\leq 40 \%$, il faudra mettre en place un CAP et titrer le traitement en fonction des objectifs suivants : - pression capillaire pulmonaire : 6 à 10 mmHg;

- index cardiaque : $>2,4 \mathrm{~L} / \mathrm{min} / \mathrm{m}^{2}$;

- RVS : 800 à 1200 dynes $/ \mathrm{s}-\mathrm{cm}^{5}$;

- ITSVL : > $15 \mathrm{~g} / \mathrm{kg}$-minute.

- Les données produites par le CAP sont pertinentes pour déterminer le traitement hémodynamique et la transplantabilité du cœur, quels que soient les résultats de l'échocardiographie.

- Envisager de répéter l'échocardiographie aux 6 à 12 h.

- Coronarographie

Indications :

- antécédents de prise de cocaïne;

- homme > 55 ans ou femme > 60 ans;

- homme > 40 ans ou femme > 45 ans présentant deux facteurs de risque ou plus;

- $\geq$ trois facteurs de risque à n'importe quel âge.

Facteurs de risque :

- tabagisme;

- hypertension;

- diabète;

- hyperlipidémie;

- indice de masse corporelle > 32;

- antécédents de la maladie dans la famille;

- antécédents de coronaropathie;

- ischémie révélée par l'électrocardiographie;

- anomalies de la mobilité de la paroi antérolatérale révélées par l'échocardiographie;

- fraction d’éjection $\leq 40 \%$, déterminée par échocardiographie bidimensionnelle.

Précautions :

- Il faut s'assurer qu'il y a normovolémie.

- Administrer de la N-acétylcystéine prophylactique à raison de 600 à 1000 mg par voie entérale deux fois par jour (première dose dès que l'angiographie l'indique) ou par voie IV à 150 mg/kg dans $500 \mathrm{~mL}$ de solution physiologique normale en 30 minutes, immédiatement avant l'agent de contraste, et ensuite $50 \mathrm{mg} / \mathrm{kg}$ dans $500 \mathrm{~mL}$ de solution physiologique normale en $4 \mathrm{~h}$.

- Il faut utiliser un agent de contraste radiologique à faible risque (non ionique, iso-osmolaire), à un volume minimum, sans ventriculographie.

Ordonnances - Radiographie pulmonaire aux $24 \mathrm{~h}$ et au besoin.

particulières aux - Bronchoscopie, coloration de Gram et culture d'un échantillon prélevé par lavage bronchique.

poumons

- Succion endotrachéale de routine, rotation en position latérale aux $2 \mathrm{~h}$.

- Valeurs cibles de la ventilation mécanique :

- Volume courant : 8 à $10 \mathrm{~mL} / \mathrm{kg}$, PFEP : $5 \mathrm{cmH}_{2} \mathrm{O}$; PIP : $\leq 30 \mathrm{~cm} \mathrm{H}_{2} \mathrm{O}$;

- $\mathrm{pH}:$ 7,35-7,45, $\mathrm{PaCO}_{2}$ : 35-45 mm Hg; $\mathrm{PaO}_{2}: \leq 80 \mathrm{mmHg}$; saturation $\mathrm{O}_{2}$ : = $95 \%$.

- Manœuvres de recrutement possibles en cas de déficit de l'oxygénation :

- Élévation périodique de la PEEP jusqu'à $15 \mathrm{~cm} \mathrm{H} \mathrm{H}_{2} \mathrm{O}$

- Inflations soutenues (PIP à $30 \mathrm{~cm} \mathrm{H} \mathrm{H}_{2} \mathrm{O}$ aux 30 à $60 \mathrm{~s}$ );

- Diurèse jusqu'à la normovolémie.

Enfants donneurs d'organes

Ces ordonnances s'appliquent aux enfants, de la naissance jusqu'à 18 ans, et portent sur des soins dispensés aux soins intensifs pédiatriques. Les posologies recommandées s'appliquent à des enfants pesant $60 \mathrm{~kg}$ ou moins; pour ceux qui pèsent plus de $60 \mathrm{~kg}$, il faut appliquer les recommandations destinées aux adultes.

Surveillance - Sonde urinaire pour assurer le drainage, contrôle strict des ingérés et des excrétés.

standard - Sonde nasogastrique pour assurer le drainage.

- Signes vitaux à l'heure.

- Oxymétrie pulsée, électrocardiographie à 3 dérivations.

- Pression veineuse centrale.

- Surveillance de la tension artérielle au moyen d'un cathéter artériel.

Analyses de

laboratoire

- Gaz sanguins artériels, électrolytes et glucose aux $4 \mathrm{~h}$ et au besoin.

- Formule sanguine complète aux $8 \mathrm{~h}$.

- Azone uréique du sang et créatinine aux $6 \mathrm{~h}$.

- Analyses d'urine.

- AST, ALT, bilirubine (totale et directe), RIN (ou temps de Quick) et temps de céphaline aux 6 h. 
Surveillance et Valeurs cibles générales : fréquence cardiaque et tension artérielle selon les plages normales pour l'âge.

traitement - Réanimation liquidienne pour maintenir une normovolémie, pression veineuse centrale de 6 à 10 mm Hg

hémodynamiques - Seuils de traitement de l'hypertension artérielle selon l'âge :

nouveau-nés de 3 mois ou moins $\quad>90 / 60 \mathrm{~mm} \mathrm{Hg}$;

> 3 à 12 mois

$>110 / 70$ mm Hg;

1 à 12 ans

>130/ 80 mm Hg;

$>12$ à 18 ans

$>140 / 90 \mathrm{~mm} \mathrm{Hg}$.

- Procéder au sevrage des agents inotropes et vasopresseurs, et commencer au besoin à administrer

- Le nitroprussiate : 0,5 à 5,0 $\mu \mathrm{g} / \mathrm{kg} / \mathrm{min}$, ou

- L'esmolol : bol de 100 à $500 \mu \mathrm{g} / \mathrm{kg}$, suivi d'une dose de 100 à $300 \mu \mathrm{g} / \mathrm{kg} / \mathrm{min}$.

- Lactate sérique aux 2 à 4 h.

- Oxymétrie veineuse centrale aux 2 à $4 \mathrm{~h}$ : titrer le traitement de façon à obtenir une saturation en oxygène du sang veineux central de $\geq 60 \%$.

Agents indiqués - Dopamine : $\leq 10 \mu \mathrm{g} / \mathrm{kg} / \mathrm{min}$.

comme traitement - Vasopressine : 0,0003 à 0,0007 U/kg/min (de 0,3 à 0,7 mU/kg/min) jusqu'à concurrence de 2,4 U/h.

de soutien

hémodynamique

Glycémie et - Perfusions systématiques de dextrose par voie intraveineuse.

nutrition - Mise en place ou maintien d'une alimentation entérale, selon la tolérance.

- Maintien d'une alimentation parentérale déjà en place.

- Mise en place et réglage d'une insulinothérapie par perfusion pour maintenir une glycémie de 4 à $8 \mathrm{mmol} / \mathrm{L}$.

Liquides et Débit urinaire : 0.5-3 mL/ $\mathrm{kg} / \mathrm{h}$.

électrolytes $\quad$ Sodium sérique : 130-150 mM.

- Taux de potassium, de calcium, de magnésium et de phosphate dans les plages normales.

Diabète insipide Caractéristiques:

- Débit urinaire $>4 \mathrm{~mL} / \mathrm{kg} / \mathrm{h}$ associé à

- Une élévation du taux de Na sérique jusqu'à $\geq 145 \mathrm{mmol} / \mathrm{l}$ et (ou)

- Une élévation de l'osmolarité sérique jusqu'à $\geq 300$ mosM et (ou)

- Une réduction de l'osmolarité urinaire jusqu'à $\leq 200 \operatorname{mos} M$

Traitement (à régler de façon à produire un débit urinaire de $\leq 3 \mathrm{~mL} / \mathrm{kg} / \mathrm{h}$ ) :

- Perfusion intraveineuse de vasopressine : 0,0003 à 0,0007 U/kg/min (de 0,3 à 0,7 mU/kg/min) jusqu’à concurrence de 2,4 U/h, et (ou)

- DDAVP intermittente : 0,25-1 $\mathrm{gg}$ IV aux $6 \mathrm{~h}$ (il n’y a pas de limite supérieure réelle de la dose; il faut titrer le traitement en fonction du débit urinaire visé).

Hormonothérapie Caractéristiques:

combinée $\quad$ Tétra-iodothyronine (T4) : bol de $20 \mu \mathrm{g}$ IV suivi d'une perfusion IV de $10 \mu \mathrm{g} / \mathrm{h}$ (ou bol de 50 à $100 \mu \mathrm{g}$ IV, suivi d'une perfusion IV de 25 à $50 \mu \mathrm{g}$ aux 12 heures).

- Vasopressine : 0,0003 à 0,0007 U/kg/min (de 0,3 à 0,7 mU/kg/min) jusqu'à concurrence de 2,4 U/h.

- Méthylprednisolone : $15 \mathrm{mg} / \mathrm{kg}(\leq 1 \mathrm{~g})$ IV aux $24 \mathrm{~h}$.

Indications :

- Échocardiographie bidimensionnelle de la fraction d'éjection $\leq 40 \%$, ou

- Instabilité hémodynamique (dont état de choc ne répondant pas au rétablissement d'une normovolémie et nécessitant un traitement vasoactif de soutien [dopamine à une dose > $10 \mu \mathrm{g} / \mathrm{min}$ ou tout agent vasopresseur]).

- Il faut envisager une hormonothérapie combinée chez tous les donneurs.

Hématologie - Hémoglobine optimale : 90-100 g/L; dans le cas des donneurs instables, la concentration la plus faible acceptable est de $70 \mathrm{~g} / \mathrm{L}$.

- Plaquettes, RIN, temps de céphaline : aucune valeur cible prédéterminée; transfusions dans les cas d'hémorragies cliniquement importantes.

- Aucune exigence particulière ne s'applique aux transfusions.

Microbiologie (de - Hémocultures quotidiennes.

référence, - Cultures d'urine quotidiennes.

quotidienne et au - Cultures quotidiennes d'échantillons prélevés par tube endotrachéal.

besoin)

- Antibiothérapie dans les cas d'infection présumée ou confirmée.

Ordonnances - Électrocardiographie à 12 dérivations.

particulières au - Troponine I ou T, aux $12 \mathrm{~h}$.

cœur

- Échocardiographie bidimensionnelle

- Seulement après une réanimation liquidienne et hémodynamique;

- Si la fraction d'éjection est de $\leq 40 \%$, répéter l'échocardiographie aux 6 à 12 h .

- Radiographie pulmonaire aux $24 \mathrm{~h}$ et au besoin.

- Coloration de Gram et culture d'un échantillon prélevé par bronchoscopie et par lavage bronchique.

- Succion de routine par tube endotrachéal, rotation en position latérale aux $2 \mathrm{~h}$.

- Valeurs cibles de la ventilation mécanique :

- Volume courant : 8-10 mL/kg, PEEP : $5 \mathrm{~cm} \mathrm{H} 2$; PIP : $\leq 30 \mathrm{~cm} \mathrm{H}_{2} \mathrm{O}$;

- $\mathrm{pH}: 7,35-7,45 ; \mathrm{PaCO}_{2}: 35-45 \mathrm{~mm} \mathrm{Hg} ; \mathrm{PaO}_{2}: \geq 80 \mathrm{~mm} \mathrm{Hg}$; saturation $\mathrm{O}_{2}: \geq 95 \%$.

- Manœuvres de recrutement possibles en cas de déficit de l'oxygénation :

- Augmentation périodique de la PEEP jusqu'à $15 \mathrm{~cm} \mathrm{H} \mathrm{H}_{2} \mathrm{O}$;

- Inflations soutenues (PIP à $30 \mathrm{~cm} \mathrm{H} \mathrm{H}_{2} \mathrm{O}$ 30-60 s);

- Diurèse à l'établissement d'une normovolumie. 
Conférenciers experts : Des conférenciers experts ont présenté des exposés détaillés qui ont joué un rôle important dans l'élaboration des recommandations présentées dans ce rapport. Ces conférenciers sont indiqués ci-dessous, dans l'ordre de leur inscription à l'ordre du jour.

Prise en charge multisystémique de donneurs de multiples organes

$D^{r}$ Sam D. Shemie (allocution sur les défis), Unité de soins intensifs pédiatriques, Hôpital de Montréal pour enfants, Centre universitaire de santé McGill, Montréal (Qc); membre honoraire du personnel, Département de médecine de soins intensifs, Hôpital pour enfants malades, Université de Toronto, Toronto (Ont.); Conseil canadien pour le don et la transplantation; Société canadienne de soins intensifs; $D^{r}$ Joe Pagliarello (principes de la prise en charge des donneurs à l'unité des soins intensifs), Directeur médical, Soins intensifs aux adultes, Programme de dons d'organes et de tissus, Hôpital d'Ottawa, Université d'Ottawa, Ottawa (Ont.); président désigné, Société canadienne de soins intensifsp; M. Kevin O’Connor (perspective des É.-U. sur la prise en charge des donneurs), Directeur des services de dons, Banque d'organes de la Nouvelle-Angleterre, Newton (Massachusetts); Dr Bruce Rosengard (perspectives internationales sur la prise en charge des donneurs : de Crystal City à Papworth); Professeur et directeur, Unité de chirurgie cardiothoracique, Hôpital Papworth, Université de Cambridge, Cambridge, Royaume-Uni; Société internationale de transplantation cardiaque et pulmonaire; American Society of Transplantation; British Heart Foundation; Dr Dimitri Novitzky (justification scientifique de l'hormonothérapie chez les donneurs d'organes, Chef, Recherche clinique, Hôpital pour anciens combattants James-A.-Haley; professeur de chirurgie cardiothoracique, Université de la Floride du Sud, Tampa (Floride); Dr Myron Kauffman (l’hormonothérapie et ses répercussions sur la transplantabilité aux Etats-Unis), Transplantation rénale, Réseau unifié de distribution d'organes, Richmond (Virginie);

\section{Considérations particulières à certains organes : cœur, poumons et organes abdominaux}

$\mathrm{D}^{r}$ Vivek Rao (panel sur le cœur), Directeur chirurgical, Transplantation cardiaque, Réseau universitaire de santé, Université de Toronto, Toronto (Ont.); Société canadienne de transplantation; Dr Shaf Keshavjee (panel sur les poumons), Directeur, Programme de transplantation pulmonaire de Toronto, Réseau universitaire de santé, Université de Toronto, Toronto (Ont.); Société canadienne de transplantation; $D^{r}$ Paul Greig (panel sur le foie); Directeur, Programme de transplantation d'organes de l'appareil digestif, Hôpital Toronto General, Université de Toronto, Toronto (Ont.); Société canadienne de transplantation; Registre canadien du remplacement d'organes; D' Sandra Cockfield (panel sur les reins), Directrice médicale, Programme de transplantation rénale, Hôpital de l’Université de l’Alberta, Université de l'Alberta, Edmonton (Alb.); Société canadienne de transplantation

\section{Autres considérations générales}

$\mathrm{M}^{\mathrm{me}}$ Kim Badovinac (utilisation d'organes provenant de donneurs cadavériques canadiens : données du Registre canadien du remplacement d'organes), Consultante, Institut canadien d'information sur la santé, Toronto (Ont.); Registre canadien du remplacement d'organes; $\mathrm{D}^{r}$ Heather Ross (revue multicentrique canadienne de l'utilisation de coeurs et de poumons), Directrice médicale, Programme de transplantation cardiaque, Réseau universitaire de santé, Toronto (Ont.); présidente, Société canadienne de transplantation

\section{Groupe des recommandations issues du Forum}

Dr Andrew Baker, Directeur médical, Unité des soins intensifs (Traumatologie et neurochirurgie), Hôpital St. Michael's, Université de Toronto, Toronto (Ont.); président, Comité consultatif clinique, Réseau Trillium pour le don de vie; Société canadienne de soins intensifs; Société canadienne des anesthésiologistes; $M^{\text {me }}$ Tracy Brand, Gestionnaire du programme provincial, Programme de transplantation de la Saskatchewan, Saskatoon (Saskatchewan); président, Association canadienne de transplantation; $D^{r}$ Christopher Doig, Unité de soins intensifs multisystémiques, Hôpital Foothills, Département des soins intensifs, Université de Calgary, Edmonton (Alb.); président, Comité des dons, Conseil canadien pour le don et la transplantation; Société canadienne de soins intensifs; Dr Paul Greig, Directeur, Programme de transplantation d'organes de l'appareil digestif, Hôpital Toronto General, Université de Toronto, Toronto (Ont.); Société canadienne de transplantation; Registre canadien du remplacement d'organes; $D^{r}$ Cameron Guest, Département de médecine de soins intensifs, Centre des sciences de la santé Sunnybrook et Women's College, Université de Toronto, Toronto (Ont.); médecin chef, Réseau Trillium pour le don de vie; Société canadienne de soins intensifs; $D^{r}$ Shaf Keshavjee, Directeur, Programme de transplantation pulmonaire de Toronto, Réseau universitaire de santé, Université de Toronto, Toronto (Ont.); Société canadienne de transplantation; Dr Peter Nickerson, Directeur du laboratoire d'immunogénétique, Société canadienne du sang,; Centre des sciences de la santé, Université du Manitoba, Winnipeg (Man.); Société canadienne de transplantation; $D^{r}$ Joe Pagliarello, Directeur médical, Soins intensifs pour adultes, Programme de dons d'organes et de tissus, Hôpital d'Ottawa, Université d'Ottawa, Ottawa (Ont.); président désigné, Société canadienne de soins intensifs; $D^{r}$ Vivek Rao, Chirurgien-chef, Programme de transplantation cardiaque, Réseau universitaire de santé, Université de Toronto, Toronto (Ont.); Société canadienne de transplantation; $D^{r}$ Heather Ross, Directrice médicale, Programme de transplantation cardiaque, Réseau universitaire de santé, Université de Toronto, Toronto (Ont.); présidente, Société canadienne de transplantation; Dr Sam D. Shemie, Unité de soins intensifs pédiatriques, Hôpital de Montréal pour enfants, Centre universitaire de santé McGill, Montréal (Qc); membre honoraire du personnel, Département de médecine de soins intensifs, Hôpital pour enfants malades, Université de Toronto, Toronto (Ont.); Conseil canadien pour le don et la transplantation; Société canadienne de soins intensifs; $M^{\text {me }}$ Kimberly Young, Analyste principale de politiques, Secrétariat du Conseil canadien pour le don et la transplantation, Santé Canada, Edmonton (Alb.); directrice de projet, Forum Optimiser le maintien du donneur afin d'accroître le nombre d'organes admissibles pour la transplantation

\section{Groupe des recommandations applicables aux enfants}

$D^{r}$ Mary Bennett, Directrice du programme de résidence, Soins intensifs pédiatriques, Hôpital de la Colombie-Britannique pour enfants, Université de la Colombie-Britannique, Vancouver (C.-B.); Société canadienne de soins intensifs; Dr David Creery, Directeur, Unité de soins intensifs pédiatriques, Centre hospitalier pour enfants de l'est de l'Ontario, Université d'Ottawa, Ottawa (Ont.); Société canadienne de soins intensifs; $D^{r}$ Anne Dipchand, Chef des services cliniques, Programme de transplantation cardiaque, Hôpital pour enfants malades, Université de Toronto, Toronto (Ont.); Société canadienne de transplantation; $D^{r}$ Catherine Farrell, Pédiatre intensiviste et présidente, Comité des dons d'organes, Unité des soins intensifs pédiatriques, Hôpital Sainte-Justine, Université de Montréal, Montréal (Qc); Société canadienne de soins intensifs; Dr Diane Hébert, Directrice des services cliniques, Programme de transplantation d'organes multiples chez enfants, Centre universitaire de santé McGill, Montréal (Qc); Association canadienne des infirmières et infirmiers en soins intensifs; $D^{r}$ Michel Lallier, Chirurgien-chef, Transplantation, Hôpital Sainte-Justine, Université de Montréal, Montréal (Qc); Société canadienne de 
Transplantation; Dr Sam D. Shemie, Division des soins intensifs pédiatriques, Hôpital de Montréal pour enfants, Centre universitaire de santé McGill, Montréal (Qc); membre honoraire du personnel, Département de médecine de soins intensifs, Hôpital pour enfants malades, Université de Toronto, Toronto (Ont.); Conseil canadien pour le don et la transplantation; Société canadienne de soins intensifs; $\mathrm{D}^{r}$ Lori West, Chef de section, Programme de transplantation cardiaque, Hôpital pour enfants malades, Université de Toronto, Toronto (Ont.); Société canadienne de transplantation; $M^{\mathrm{me}}$ Kimberly Young, Analyste principale des programmes, Secrétariat du Conseil canadien pour le don et la transplantation, Santé Canada, Edmonton (Alb.); directrice du Forum Optimiser le maintien du donneur afin d'accroître le nombre d'organes admissibles pour la transplantation

\section{Logistique et application des connaissances}

Deborah Gordon (présidente), Chef principal de l'exploitation, Santé de la capitale; Hôpital de l'Université de l'Alberta, Edmonton (Alb.); $M^{\text {me }}$ Kim Badovinac, Consultante, Institut canadien d'information sur la santé, Toronto (Ont.); Registre canadien du remplacement d’organes; M. Bill Barrable, Directeur général provincial, Société de transplantation et Institut de recherche de la Colombie-Britannique, Vancouver (C.-B.); $M^{\text {me }}$ Mance Cléroux, Directrice générale, Québec-Transplant, Montréal (Qc); Association canadienne de transplantation; M. Thorsten Duebel, Directeur par intérim, Secrétariat du Conseil canadien pour le don et la transplantation, Edmonton (Alb.); ${ }^{\text {me }}$ Nora Johnston, Directrice d'équipe de projet, Santé et Mieux-être Alberta, Edmonton (Alb.); M. Darwin Kealey, Président et chef de la direction, Réseau Trillium pour le don de vie, Toronto (Ont.); $M^{\text {me }}$ Joyce MacMullen, Gestionnaire de service de santé, Soins intensifs et coordonnatrice des dons d'organes, Centre des sciences de la santé Queen Elizabeth II, Halifax (N.-É.); Association canadienne des infirmières et infirmiers en soins intensifs; $M$. Burton Mattice, Vice-président et chef de l'exploitation, Services de donneurs de la Caroline, Durham (Caroline du Nord); présidente, Association of Organ Procurement Organization Procurement Council; $M^{\mathrm{me}}$ Ann Secord, Responsable des dons d’organes, Direction des services de santé, Santé et Mieux-être Nouveau-Brunswick, Fredericton (N.-B.)

\section{Autres participants}

Dana Baran, Directrice médicale, Québec-Transplant, Néphrologie de la transplantation; Centre universitaire de santé McGill, Montréal (Qc); Société canadienne de transplantation; Jeffrey Barkun, Transplantation hépatique, Centre universitaire de santé McGill, Université McGill, Montréal (Qc); Société canadienne de transplantation; Stephen Beed, Directeur des soins intensifs, Centre des sciences de la santé Queen Elizabeth II, Université Dalhousie, Halifax (N.-É.); Société canadienne de soins intensifs; Société canadienne des anesthésiologistes; Karen Burns, Centre des sciences de la santé de London, Hôpital Victoria, Programme de méthodologie de la recherche en santé, Université McMaster, Hamilton (Ont.); Société canadienne de soins intensifs; clinicienne chercheure en soins intensifs; David Clarke, Département de neurochirurgie, Centre des sciences de la santé Queen Elizabeth II , Halifax (N.-É); Société canadienne de neurochirurgie; Rosemary Craen, Directrice, Département de neuroanesthésie, Centre des sciences de la santé de London , Université Western Ontario, London (Ont.); Société canadienne des anesthésiologistes; Marc Deschênes, Directeur médical, Transplantation hépatique, Centre universitaire de santé McGill, Montréal (Qc); Société canadienne de transplantation; Jan Emerton, Spécialiste des dons d'organes, Société de transplantation de la Colombie-Britannique, Vancouver (C.-B.); Association canadienne de transplantation; David Forrest, Directeur associé (suppléant), Soins intensifs, Hôpital Vancouver General, Vancouver (C.-B.); Société canadienne de soins intensifs; Peter Goldberg, Directeur, Services des soins intensifs, Hôpital Royal Victoria, Centre universitaire de santé McGill, Montréal (Qc); Société canadienne de soins intensifs; Peter Gorman, Programme de dons d'organes et de tissus du Nouveau-Brunswick - Comité directeur du programme, Département de neurochirurgie, Régie de la santé du Sud-Est, Moncton (N.-B); Mark Heule, Directeur des Soins intensifs, Hôpital Misericordia, Edmonton (Alb.); Société canadienne de soins intensifs; Dan Howes, Hôpital général de Kingston, Université Queen's, Kingston (Ont.); Association canadienne des médecins d'urgence; James Kutsogiannis, Médecin-chef des dons, Santé de la capitale, Université de l'Alberta, Edmonton (Alb.); Société canadienne de soins intensifs; Stéphan Langevin, Hôpital de l’Enfant-Jésus, Université Laval, Québec (Qc); Société canadienne de soins intensifs, Société des intensivistes du Québec; Joseph Lawen, Chirurgien-chef, Programme de transplantation rénale, Centre des sciences de la santé Queen Elizabeth II, Halifax (N.-É.); Société canadienne de transplantation; Neil Lazar, Directeur général, Hôpital Toronto General, Unité de soins intensifs chirurgicaux médicaux, Soins intensifs aux adultes, Réseau universitaire de santé, Université de Toronto, Toronto (Ont.); Société canadienne de soins intensifs; Michelle Leahey, Soins intensifs et coordonnatrice des dons d'organes, Centre des sciences de la santé Queen Elizabeth II , Halifax (N.-É.); Association canadienne de transplantation; Marie-Hélène LeBlanc, Directrice médicale, Insuffisance cardiaque et transplantation cardiaque, Hôpital Laval, Université Laval, Sainte-Foy (Qc); Société canadienne de transplantation; Dale Lien, Directeur médical, Programme de transplantation pulmonaire, Hôpital de l'Université de l'Alberta, Université de l'Alberta, Edmonton (Alb.); Société canadienne de transplantation; Karen McRae, Directrice des anesthésies pour les chirurgies thoraciques et les transplantations pulmonaires, Réseau universitaire de santé, Toronto (Ont.); Société canadienne des anesthésiologistes; Joy Mintenko, Soins intensifs chirurgicaux, médicaux et pédiatriques, Hôpital général de Regina, Regina (Sask.); Association canadienne des infirmières et infirmiers en soins intensifs; Terrance Myles, Société canadienne de neurochirurgie; directeur par intérim de la Division de neurochirurgie, Faculté de médecine, Université de Calgary (Alberta); Clare Payne, Association canadienne de transplantation; gestionnaire des services cliniques, Réseau Trillium Gift of Life Network, Toronto (Ont.); Sharon Peters, Société canadienne de soins intensifs; Soins intensifs aux adultes, Corporation des soins de santé de St. John's, St. John's (T.-N.-L.); Ann Secord, responsable de l'obtention des organes, Direction des services de santé, ministère de la Santé et du Mieux-être du Nouveau-Brunswick, Fredericton (N.-B.); Michael Sharpe, Société canadienne de soins intensifs; Soins intensifs aux adultes, Centre des sciences de la santé de London. London (Ont.); Susan Shaw, Société canadienne de soins intensifs; Société canadienne des anesthésiologistes; directrice médicale, Unité des soins intensifs, hôpital Royal University, Saskatoon (Sask.); Ken Stewart, Société canadienne de transplantation; chirurgien en transplantation des poumons, hôpital de l'Université de l'Alberta, Edmonton (Alb.); Bernard Tremblay, Association canadienne de transplantation; coordonnateur des acquisitions cliniques, Québec-Transplant; infirmier clinicien en dons d'organes et de tissus, Centre de santé de l'Université McGill, Montréal (Qc); Helmut Unruh, Société canadienne de transplantation; directrice de programme, Manitoba Lung Transplant, Centre des sciences de la santé de l'Université du Manitoba, Winnipeg (Man.); Corinne Weernink, présidente désignée, Association canadienne de transplantation; coordonnatrice des donneurs d'organes, Centre des sciences de la santé de London, London (Ont.); Bryan Young, Canadian Neurocritical Care Group; Division de neurologie et de médecine des soins intensifs, Centre des sciences de la santé de London, London (Ont.)

\section{Consultants}

Strachan-Tomlinson and Associates, Ottawa, (Ont.); Animation : Dorothy Strachan; Recherche : Paul Tomlinson; Gestion de projet et rapports : Peter Ashley et Lisa Weiss 